Fall 2016

\title{
Yeast Dynamin Associates With The Garp Tethering Complex For Endosome-To-Golgi Traffic
}

Uma Saimani

As with any intellectual project, the content and views expressed in this thesis may be considered objectionable by some readers. However, this student-scholar's work has been judged to have academic value by the student's thesis committee members trained in the discipline. The content and views expressed in this thesis are those of the student-scholar and are not endorsed by Missouri State University, its Graduate College, or its employees.

Follow this and additional works at: https://bearworks.missouristate.edu/theses

Part of the Biology Commons

\section{Recommended Citation}

Saimani, Uma, "Yeast Dynamin Associates With The Garp Tethering Complex For Endosome-To-Golgi Traffic" (2016). MSU Graduate Theses. 3043.

https://bearworks.missouristate.edu/theses/3043

This article or document was made available through BearWorks, the institutional repository of Missouri State University. The work contained in it may be protected by copyright and require permission of the copyright holder for reuse or redistribution.

For more information, please contact BearWorks@library.missouristate.edu. 


\title{
YEAST DYNAMIN ASSOCIATES WITH THE GARP TETHERING COMPLEX FOR ENDOSOME-TO-GOLGI TRAFFIC
}

\author{
A Masters Thesis \\ Presented to \\ The Graduate College of \\ Missouri State University \\ In Partial Fulfillment \\ Of the Requirements for the Degree \\ Master of Science, Biology
}

\author{
By \\ Uma Saimani \\ December 2016
}


Copyright 2016 by Uma Saimani 


\title{
YEAST DYNAMIN ASSOCIATES WITH THE GARP TETHERING COMPLEX \\ FOR ENDOSOME-TO-GOLGI TRAFFIC
}

\section{Biology}

Missouri State University, December 2016

Master of Science

Uma Saimani

\begin{abstract}
Yeast dynamin, Vacuolar Protein Sorting 1 (Vps1), has been implicated in recycling proteins and lipids from the endosome to the trans-Golgi network (TGN). Previous research showed a genetic interaction of Vps1 with all components of the GARP tethering complex, which anchors vesicles at the late Golgi membrane. I used the yeast two-hybrid system and pinpointed a 33 amino acid segment of Vps51, a GARP subunit, that interacts with Vps1. Based on sequence homology between Vps51 and its mammalian homolog ANG2 in the 33 amino acid stretch, I identified two key residues of Vps51, E127, and Y129, that bind Vps1. The replacement of these residues led to severe defects in endosome-to-TGN transport of Snc1, providing evidence of the physiological relevance of the interaction of Vps51 with Vps1 via these residues for the traffic.

Furthermore, my functional analysis revealed that Vps1 acts upstream of Vps51, and the absence of Vps1 resulted in reduced localization levels of Vps51 and its binding partner Tlg1 to the late Golgi. Taken together, I propose that Vps1 functions with the GARP tethering machinery for efficient tethering/fusion at the TGN.
\end{abstract}

KEYWORDS: Vps1, Vps51, retrograde pathway, endosome, TGN, GARP, yeast

This abstract is approved as to form and content

Dr. Kyoungtae Kim
Chairperson, Advisory Committee
Missouri State University 


\title{
YEAST DYNAMIN ASSOCIATES WITH THE GARP TETHERING COMPLEX FOR ENDOSOME-TO-GOLGI TRAFFIC
}

\author{
By \\ Uma Saimani \\ A Masters Thesis \\ Submitted to the Graduate College \\ Of Missouri State University \\ In Partial Fulfillment of the Requirements \\ For the Degree of Master of Science, Biology
}

December 2016

Approved:

Kyoungtae Kim, $\mathrm{PhD}$

Paul Durham, PhD

Ryan Udan, PhD

Julie Masterson, PhD: Dean, Graduate College 


\section{ACKNOWLEDGEMENTS}

First and foremost, I wish to express my sincerest gratitude to my committee chair Dr. Kyoungtae Kim for his eternal optimism and able guidance that helped me accomplish my aims. I also want to thank my committee members, Dr. Paul Durham and Dr. Ryan Udan for their valuable time and constant encouragement throughout my time here at Missouri State University. Additionally, I would like to thank my colleagues Hyoeun McDermott, Christopher Trousdale, Shiva Kumar Gadila, Bryan Banh, Pelin Makaraci, Sara Woodman, Mariel Delgado Cruz, John Short, Ashley Smock, and Jared Smothers for their unconditional support. Next, I wish to thank my family and my friends in Springfield for helping me out when most needed. Finally, I would like to acknowledge the Biology Department and the Graduate College for their financial support.

I dedicate this thesis to my parents. 


\section{TABLE OF CONTENTS}

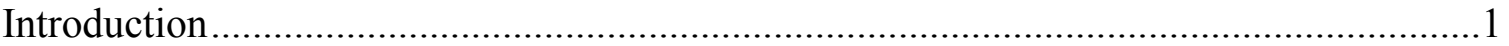

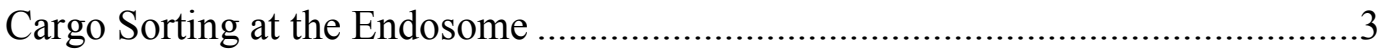

Fission and Movement of the Transport Carrier towards the TGN .........................

Tethering at the TGN ...............................................................................10

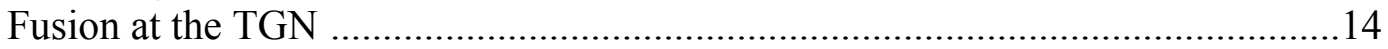

Problem Statement and Hypothesis ....................................................................16

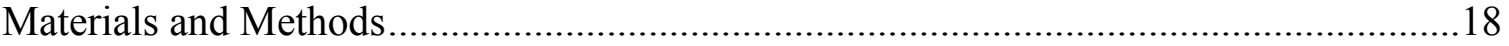

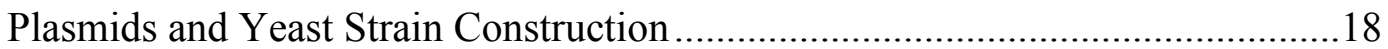

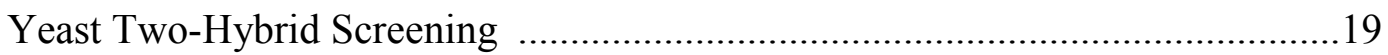

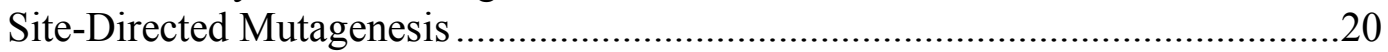

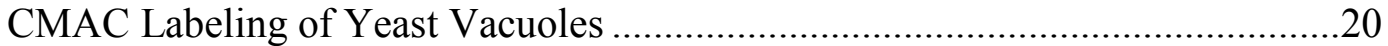

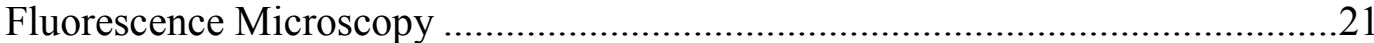

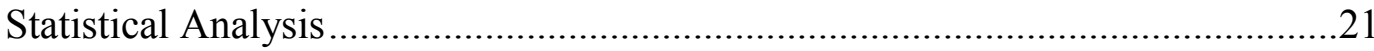

Bioinformatics Tools for Sequence Homology and Homology Modeling ............21

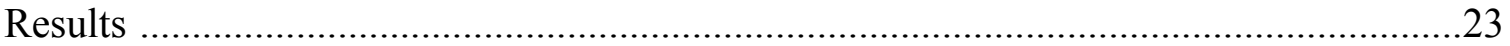

Yeast Two-Hybrid Interaction between Vps1 and Vps51 ……...........................23

Two Residues, E127 and Y129 of Vps51 are implicated in its Interaction with Vps1 ...........................................................................24

Vps51 Colocalizes with Vps1 and the Loss of Vps1 Affects Localization of Vps51 and Tlg1 at the Late Golgi.

The Functional Relationship between Vps1 and Vps51 during Tethering/Fusion

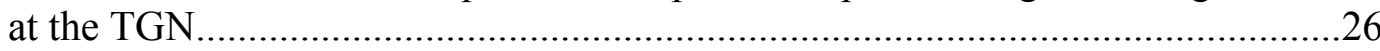

Physiological Significance of Vps1-Vps51 Interaction.........................................27

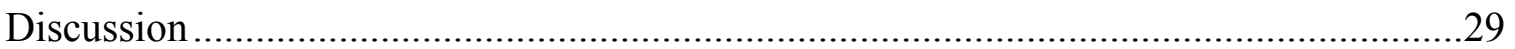

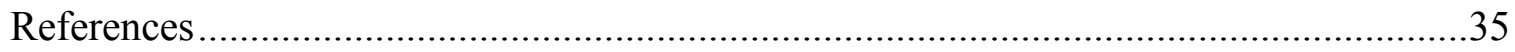




\section{LIST OF TABLES}

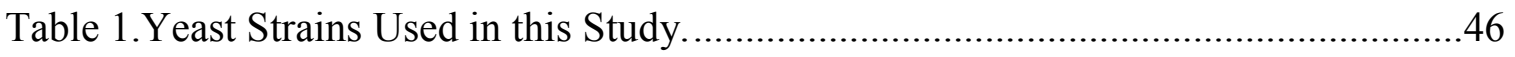

Table 2. Bacterial Plasmids Used in this Study ........................................................49 


\section{LIST OF FIGURES}

Figure 1. Retrograde Transport from the Endosome to the TGN .................................51

Figure 2. Characterization of the Interaction between Vps1 and Vps51 ........................52

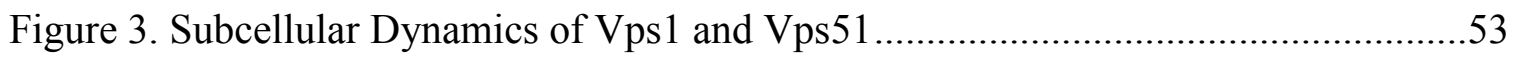

Figure 4. Vps1 and Vps51 Collaborate for Retrograde Transport of $v$-SNARE Snc1 „.....54

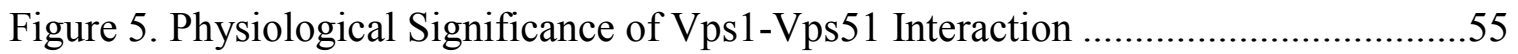

Figure 6. Effect of Point Mutations on Localization of Vps51 ....................................56

Figure 7. Model for Tethering/Fusion at the TGN ....................................................57 


\section{INTRODUCTION}

Eukaryotic cells have many membrane-bound organelles. Constant communication and exchange of essential proteins and lipids occur between these compartments via intracellular trafficking, essential for cellular homeostasis, signal transduction, and cell viability (Tokarev et al., 2009; Watson et al., 2005). In the last few decades, much attention has been given to understanding intracellular trafficking. This has contributed to an explosive growth in unfolding discrete stages of traffic and identifying molecules that regulate each stage of transport, which has shed light on a mechanistic understanding of intracellular trafficking. For example, a novel lipid $\mathrm{PI}(3,4) \mathrm{P}_{2}$ (phosphatidylinositol-3,4-bisphosphate) was recently identified at the plasma membrane aiding scission and late stages of endocytosis (Posor et al., 2013). This disproves the notion that $\mathrm{PI}(4,5) \mathrm{P}_{2}$ (phosphatidylinositol-4,5-bisphosphate) is the only main phospholipid that regulates endocytosis at the plasma membrane. Emerging information about dynein, a microtubule minus-end directed motor, has been contradicting the traditional model which states that a single microtubule plus-end kinesin motor exerts around six times the force exerted by a dynein monomer necessitating multiple dynein monomers to come together for the cytoskeletal tug-of-war against a kinesin molecule. The new evidence shows that a single dynein forms a complex by interacting with two other non-motor proteins to win the tug-of-war (Belyy et al., 2016a). Results from another study revealed that a novel cytosolic protein, Cornichon-1, interacts with transmembrane ER cargo and allows the cargo to be packaged into Golgi-bound vesicles. The discovery has resulted in a deeper 
understanding of protein packaging at the ER (Zhang and Schekman, 2016). Such rapid and significant advancement has been possible because of accessibility to cutting edge technology, including multiphoton live cell imaging in conjunction with cell perfusion systems that has helped achieve remarkable progress in deciphering the spatiotemporal dynamics of proteins involved in intracellular trafficking (Atencia and Beebe, 2005;

Huang et al., 2015; Tie et al., 2016). However, a limitation of multiphoton imaging is that its resolution is not sufficient for studying subcellular dynamics of proteins engaged in trafficking. This caveat can be overcome by using correlative light and electron microscopy, which provides high-resolution imaging of fluorescently-labeled proteins allowing us to capture protein dynamics during trafficking (de Boer et al., 2015). Another high resolution microscopy technique is STED (Super-resolution optical stimulated emission depletion microscopy). STED has very recently been used to decipher the compartmentalization of proteins found within the sub-cellular peroxisome in human cells (Galiani et al., 2016). An alternative genetic technique, high-throughput small interfering RNA (siRNA) screen is becoming an increasingly popular tool to identify novel components and regulators involved in intracellular trafficking (Anitei et al., 2014). Intracellular trafficking is a broad term which describes a diverse spectrum of transport routes within a cell that include endocytosis by which extracellular matters and its own membrane receptors are internalized. Endocytosed cargo is targeted to the early endosome from where it follows multiple routes depending on its sorting signals (Besterman and Low, 1983; Du Toit, 2015). Certain cargoes are rapidly recycled from the early endosome to the plasma membrane directly by the fast recycling pathway, or they follow the slow recycling route wherein cargo has to pass through the recycling 
endosome before reaching the plasma membrane (Hao and Maxfield, 2000). The early endosome matures into the late endosome, and cargo from the early or late endosome are targeted to the trans-Golgi network (TGN) via the retrograde route (Mallard et al., 1998; Rohn et al., 2000). The late endosome eventually fuses with the lysosome to deliver cargo for degradation (Bohley, 1995). Lastly, secretory proteins synthesized in the ER pass through the Golgi complex and then are exocytosed via the biosynthetic/exocytic route (Rothman and Orci, 1992).

Dysregulation of the components involved in endosome-to-TGN transport is associated with various human diseases. For example, dysfunction of cargo sorting machinery at the endosome is associated with Parkinson's disease (Follett et al., 2016), as well as Alzheimer's disease (Kamagata et al., 2009; Kollmann et al., 2010; Muhammad et al., 2008; Willnow and Andersen, 2013). Therefore, a complete understanding of the molecular mechanism of the retrograde traffic is needed and is of significant biomedical importance. This review aims to provide up-to-date information pertaining to retrograde transport from the endosomal system to the TGN, and will be divided into the following four sections: 1) cargo sorting at the endosome; 2) fission and movement of transport carrier towards the TGN; 3) tethering at the TGN; and 4) fusion at the TGN.

\section{Cargo Sorting at the Endosome}

Recent studies have revealed that the early endosome is, in fact, a network comprised of two main microdomains including the Tubular Endosomal Network (TEN) and Vacuolar domain (Bonifacino and Rojas, 2006a). The latter is a hub for endosome maturation and formation of Intraluminal vesicles (ILVs), which are small vesicles found 
in the lumen of the late endosome. The matured, late endosome eventually fuses with the lysosome. The cellular processes of endosome maturation and endolysosomal degradation pathway are discussed in great depth in an article by Curtiss et al. (2007). TEN is comprised of highly tubulated subdomains harboring multiple cargo exit sites. The extension of endosomal membrane with high surface area to lumenal volume ratio makes TEN ideal for exporting cargo (Bonifacino and Rojas, 2006b; Burd, 2011). Results from a recent study have shown that the ER has cargo exit sites that don't coincide with its cargo arrival sites (Ackema et al., 2013). The possibility of TEN also having such exclusive sites for cargo import and cargo export opens up an interesting avenue for future research.

A select group of cargo travel from the endosome to the TGN, including mannose-6-phosphate receptors (MPR), Soluble N-ethylmaleimide-sensitive factor activating protein receptors (SNAREs), toxins, and other proteins that require continuous retrieval to the TGN for their function. Proper sorting of these cargo at the endosome is essential for the successful endosome-to-Golgi traffic, demanding coat proteins, adaptors, and sorting nexins (Kornfeld, 1992; Pfeffer, 2011). A well-studied coat protein-adaptor pair is clathrin and AP-1. Expression of a mutated version of clathrin or AP-1 resulted in impaired retrieval of the B subunit of Cholera toxin $(C t x B)$ from the early endosomes to the TGN (Matsudaira et al., 2015). AP-1 and clathrin also assist sorting of another wellknown cargo, MPR, from the late endosome to the TGN. There are two variants of MPR: cation-dependent MPR (CD-MPR) and cation-independent MPR (CI-MPR), of which CD-MPR utilizes clathrin and AP-1 for its transport (Schweizer et al., 2000). AP-1 recognizes a di-leucine (D/ExxxLL/I, D-Aspartate, E-Glutamate, x-any amino acid, L- 
leucine, and I-Isoleucine) and a tyrosine (YxxØ, Y-tyrosine and Ø-any hydrophobic amino acid) sorting motifs on the cytosolic face of the cargo CD-MPR, and then recruits the coat protein clathrin. CD-MPR has an additional Tyr-Phe motif in its cytoplasmic tail that allows it to be recognized by AP-1 and sorted by clathrin. Clathrin polymerizes to form a protein coat around the extending endosomal tubule, that can then be pinched off by dynamin, whose physiological role will be discussed in the section titled 'Fission and transport to the TGN' (Moore et al., 1987; Progida and Bakke, 2016). AP-1 is primarily known to work at the Golgi, but recent evidence indicates that it also works at the endosome and the plasma membrane. While the plasma membrane has an abundant amount of PI $(4,5) \mathrm{P}$, the endosomal and Golgi membranes comprise PI3P (phosphatidyl inositol-3-phosphate) and PI4P (phosphatidyl inositol-4-phosphate), respectively (Balla, 2016; van Meer and de Kroon, 2011; van Meer et al., 2008). Importantly, AP-1 has an intrinsic ability to bind to these phospholipids, allowing it to be recruited to and function at there. Regardless of its subcellular location, AP-1, a heterotetrameric adaptor composed of $\gamma, \beta 1, \mu 2$, and $\sigma 1$ subunits (Lee et al., 2008), universally recognizes the dileucine or tyrosine motifs via a selective interaction of its subunit with its cargo. For example, the $\mu 1 \mathrm{~A}$ subunit of AP-1 recognizes the tyrosine motif in the cytosolic face of cargo at the Golgi membrane (Bonifacino and Dell'Angelica, 1999), while its $\gamma / \sigma 1$ hemicoplex binds the dileucine motif (Traub, 2005). However, the question of whether these subunits of AP-1 recognize, in a similar manner mentioned above, the aforementioned cargo loaded at the endosome remains elusive.

Another well-known coat protein working at the endosome is the retromer. The retromer was first identified in yeast, and it is functionally dissected into two major 
subcomplexes: the dimeric SNX-BAR subcomplex including sorting nexins Vps5 and Vps17, and the trimeric cargo-recognition subcomplex composed of Vps26, Vps29, and Vps35 (Seaman et al., 1998). The cargo recognition subcomplex is conserved in mammals, but there is a slight divergence with the SNX-BAR subcomplex. The dimeric SNX-BAR subcomplex in mammalian cells comprises a heterodimer of sorting nexin-1 (SNX1) or SNX2 pairing up with either SNX5 or SNX6 (Collins, 2008). The sorting nexins are recruited to the endosome by the interaction of their Phox (Phagocyte Oxidase) homology domain with PI3P found in the endosomal membrane (Zhong et al., 2002). The cargo recognition subcomplex is recruited by the interaction of Rab7, a small GTPase, with VPS35 (F1A), and the interaction is stabilized by SNX3. RNAi studies have revealed that the recruitment of the two retromer subcomplexes occurs concurrently and does not depend on each other (Nisar et al., 2010; Seaman, 2012) (F1B). The cytosolic domain of most retrograde cargo has many hydrophobic and aromatic residues that can be recognized by Vps35. For example, the retromer recognizes the WLM (Wtryptophan, L-leucine, M-methionine) motif on the cytosolic face of CI-MPR, and sorts it into emerging tubules (McGough and Cullen, 2011; Vardarajan et al., 2012). Vps10, the yeast orthologue of MPR is also sorted by the retromer into tubules at the endosome (Seaman et al., 1998). Once the retromer has helped sort the cargo and extend the cargoladen tubule, it recruits additional factors like WASH, which facilitates fission and subsequent transport of cargo carrier (Harrison et al., 2014). The action mechanism of WASH is described in detail in the section titled 'Fission and Movement of transport carrier towards TGN'. 
Both clathrin and the retromer are implicated in transporting the B subunit of Shiga toxin StxB from the endosome toward the TGN (Popoff et al., 2009). Consequently, the question of whether they function in parallel or sequential transport steps arose. In a parallel relationship, loss or depletion of a coat system would be compensated by the other. However, due to the finding that severe phenotypic defects in retrograde traffic of $S t x B$ upon si-RNA knockdown of $V P S 26$ was similar to that seen in clathrin-depleted cells, the sequential transport step model was favored (Popoff et al., 2007). A recent study further strengthened the idea of a sequential transport event by revealing that the retromer function remains unaffected by clathrin depletion and that SNX-decorated tubules are not associated with clathrin (Borner et al., 2012; McGough and Cullen, 2013a). The current model of StxB retrieval event postulates that clathrin functions in cargo enrichment before recruitment of the retromer. Then, the retromer complex is assembled near the clathrin-coated region and generates cargo-enriched tubules independently of clathrin (McGough and Cullen, 2013b). Therefore, this model is consistent with the general notion that clathrin performs sorting at the early endosome and retromer at the late endosome (Pfeffer, 2011).

The need for multiple coat proteins like clathrin and the retromer at the endosome is possibly due to the diversity of cargo, departing the endosome. Indeed there are distinct subpopulations of cargo-carrying vesicles emerging from the endosome (Bonifacino and Rojas, 2006b), because of this, one cannot exclude the possibility that there could be other unidentified coat protein(s) functioning for their corresponding cargo destined for the TGN. 


\section{Fission and Movement of Transport Carrier towards the TGN.}

As the tubule is extending, VPS35 recruits the WASH (Wiskott-Aldrich protein and SCAR Homolog) complex, a type I actin nucleation promoting factor (Rotty et al., 2013), by interacting with the 'tail' domain of WASH subunit Fam21 (F1C) (Harbour et al., 2012). The WASH complex stimulates Arp2/3-mediated actin polymerization near the neck of the extending tubule. The actin polymers force the emerging tubule from the endosome to stretch toward the cytosol (F1D) (Seaman et al., 2013). Additionally, actin polymerization is also known to promote scission of the invaginating tubule because depletion of WASH results in the formation of exaggerated endosomal tubules, a phenotype reminiscent of loss of a pinchase (Derivery et al., 2009; Gautreau et al., 2014; Seaman et al., 2013). In addition to stimulating actin polymerization, the WASH complex is implicated in regulating scission of endosomal tubules through the interaction with dynamin via its subunit Fam21 (Derivery et al., 2009). Dynamin monomers self-assemble to form an oligomer that wraps around the neck of the invaginating tubule and pinches it off by GTP hydrolysis (F1D) (Antonny et al., 2016; Seaman, 2012). The contribution of dynamin in endosome-to-TGN traffic by exerting its GTP hydrolysis was first described in HeLa cells expressing dyn ${ }^{\mathrm{K} 44 \mathrm{~A}}$ mutant variant. These cells showed proper clathrinmediated endocytosis but defective retrieval of the plant toxin ricin from the endosome (Llorente et al., 1998). This result is surprising because the dyn ${ }^{\mathrm{K} 44 \mathrm{~A}}$ mutation in its Gdomain has been known to disrupt the GTP-binding/hydrolysis activity required for endocytosis (Damke et al., 2001; Kühling and Schelhaas, 2014). The question of whether this aberrant retrieval phenotype caused by the dynamin mutant is cell-type specific remains unknown. Similarly, two other studies found that both CI-MPR and STXB also 
accumulated in endosomal tubules and were not trafficked to the TGN on expression of the same dynamin mutant, which supports the role of dynamin as a pinchase at the endosome (Lauvrak et al., 2004; Nicoziani et al., 2000). Apart from these cargoes, dynamin has also recently been implicated in the transport of a viral protein, DENV-2, during retrograde trafficking (Shrivastava et al., 2011). In another study, Mesaki et al. (2011) reported a block in endosomal fission and motility but not in the tubulation process when HeLa cells were treated with the dynamin inhibitor dynasore. Taken together, all these results provide evidence to delineate the specific role for dynamin in fission at the endosome (Ferguson and De Camilli, 2012). Vps1 is a dynamin-like protein in yeast, and electron tomography study revealed that $v p s 1 \Delta$ cells have atypically elongated tubules from the endosome, similar to the tubules seen with expression of a mutated dynamin in mammalian cells. This observation confirmed that Vps1 functions as a pinchase at the endosome in yeast (Chi et al., 2014).

As tubule scission is close to completion, SNX6 interacts with the p150-glued subunit of dynactin which is in association with dynein, a microtubule minus-end directed motor. The dynein-dynactin motor will drag the transport carrier along microtubule tracks towards a non-centrosomal microtubule population near the TGN (F1E) (Hong et al., 2009). As previously mentioned, a recent discovery demonstrated that dynein conjugates with other proteins including dynactin and BicaudalD2, an adaptor protein, to exert the same force as a kinesin monomer. The study utilized an in vitro tugof-war assay and observed that the combined force exerted by dynein-dynactinBicaudalD2 is sufficient to shift the balance of forces against a single kinesin motor (Belyy et al., 2016b). However, this conclusion was made using an artificial in vitro 
assay, necessitating further study to confirm whether this dynein-associated complex works in vivo. Since this study only utilizes certain artificial cargo and the adaptor protein BicaudalD2. It will be interesting to see if dynein can exert the same force when coupled with different adaptor proteins or different cargo.

\section{Tethering at the TGN}

Once the endosome-derived transport carrier reaches the proximity of the TGN, the carrier-Golgi interaction is mediated by tethers that anchor the cargo-laden transport carrier to the target membrane. There are multiple tethers working at the TGN: the COG (Conserved Oligomeric Golgi) complex, TRAPPII (Transport Protein Particle) complex, Golgin, and the GARP (Golgi Associated Retrograde Proteins) complex. Among them, Golgin and the GARP complex are the major protein complexes involved in endosometo-TGN retrograde transport (Whyte and Munro, 2002). The COG complex and TRAPPII complex have also been implicated in retrograde transport, but they mainly function for tethering in intra-Golgi transport (Sacher et al., 1998; Ungar et al., 2002; Yip et al., 2010; Zolov and Lupashin, 2005).

The Golgin family of tethers, which are highly conserved across eukaryotes, is the only homodimeric tether sharing a coiled-coil structure. The tethering specificity of diverse subsets of Golgins was confirmed using a 'Knock-Sideways' approach, wherein different Golgins were engineered to be mistargeted to mitochondria instead of the TGN to check if the corresponding cargo is also mislocalized to mitochondria (Robinson et al., 2010). Among Golgins tested with this approach, mislocalization of the GRIP (Golgin97, RanBP2alpha, Imh1p, and p230/golgin-245) domain Golgins and TMF (TATA 
element- Modulatory Factor) led to an accumulation of common retrograde cargoes, including CD-MPR, $S T x B$, and SNARE Vtila, at the mitochondria (Fridmann-Sirkis et al., 2004; Sztul and Lupashin, 2006; Wong and Munro, 2014). The aforementioned Golgins are anchored at the TGN by interacting with the transmembrane GTPase Arl1. Furthermore, Golgins interact directly with the microtubule track or with proteins associated with the microtubules to provide a direct path for incoming transport carrier to the TGN (Efimov et al., 2007). For example, GRIP domain Golgin GCC185 binds to the microtubule regulator CLASP (cytoplasmic linker associated proteins), and helps stabilize the microtubules found near the TGN (Miller et al., 2009). As the transport carrier approaches the TGN on the microtubule track, GCC185 projects itself into the cytosol to capture the incoming vesicle by interacting with GTP-bound Rab6a found on the transport carrier membrane. This projection is possible because of the coiled-coil domains that are found along the length of the Golgins are interspersed with proline residues which act as hinges, providing the Golgins with ample flexibility to stretch and capture transport carriers easily (Cheung and Pfeffer, 2016; Sohda et al., 1994; Witkos and Lowe).

Besides Rab6a, GCC185 can bind to at least 14 other Rab proteins (Derby et al., 2007). The molecular basis for all these interactions is not clear. However, there is no substantial proof pertaining to the interaction of the other GRIP domain Golgins (golgin97, golgin-245, and GCC88) with their corresponding Rab, and this will be a lucrative avenue to understand the general functioning of the GRIP domain Golgins. Taken together, tethering at the TGN adds another layer of complexity to the alreadycomplicated process of the retrograde traffic. Due to the ever-increasing number and 
diversity of cargo, the control options are also increasing. Other than tethering, Golgins also seem to contribute to maintaining Golgi stack morphology. A comprehensive siRNA screen revealed that most of the GCC185-interacting Rabs are Golgi residents, and cells depleted of GCC185 displayed a fragmented Golgi phenotype. This phenotype was rescued when GCC185 was reintroduced into those cells. Taken together, these observations helped generate a model wherein GCC185 helps in maintaining Golgi stack morphology by interacting with the Rabs present on different stacks and linking those stacks together (Hayes et al., 2009).

The GARP is a heterotetrameric tethering complex that was initially discovered in yeast, and consists of four subunits Vps51, Vps52, Vps53, and Vps54 (Siniossoglou and Pelham, 2002a). Its mammalian counterpart consists of the latter three subunits and Ang2, the mammalian homolog of Vps51 (Perez-Victoria et al., 2010). Vps52 interacts with GTP-bound Rab6a on the transport carrier, and Vps53 interacts with Arl5 on the TGN (F1F). Therefore, the GARP complex acts as an excellent tether between the incoming transport carrier and the TGN. Loss of Rab6a resulted in partial dispersal of the GARP tether, whereas depletion of Arl5 did not disturb the GARP tether, thereby suggesting that Rab6a might be an important player for proper targeting of the GARP complex to the TGN (Rosa-Ferreira et al., 2015; Siniossoglou and Pelham, 2002a; Whyte and Munro, 2002). Recently, a novel protein TSSC1 was identified and found to interact directly with the GARP tether for efficient recruitment of the tether to the TGN. Additionally, TSSC1 was also shown to interact with the EARP (Endosome-Associated Recycling Protein) tether, which functions at recycling endosomes and the TGN (Gershlick et al., 2016). The EARP and GARP tethers share three subunits but differ at 
the fourth subunit. While GARP has Vps54 as its fourth subunit, EARP consists of syndetin (Schindler et al., 2015). An interesting question of whether TSSC1 works independently or in collaboration with Rab6a for recruiting the GARP tether arises now and needs further exploration. The GARP tether is known to interact with the TGN SNARE Syntaxin6/Tlg1 via the LxxYY (L-Leucine, $x$-any amino acid, Y-tyrosine) motif on Ang2, and a depletion of GARP subunits leads to reduced formation of the transSNARE complex at the TGN (Abascal-Palacios et al., 2013; Fridmann-Sirkis et al., 2006). Deletion of any GARP subunit impairs retrograde transport of CI-MPR, STxB, yeast Kex2, and many other cargoes, suggesting that the GARP tether participates in transport from both the early endosome and late endosome (Perez-Victoria et al., 2008). As previously mentioned, Golgins interact with the microtubule track to provide the incoming transport carrier with a direct path to the TGN. However, there is no documented evidence yet supporting the GARP tether's interaction with microtubules or with the transport carrier itself through membrane lipids (Cheung and Pfeffer, 2016). Understanding this will provide a more complete model of the GARP tether's mode of action in vivo.

Furthermore, the GARP complex seems to be essential for bidirectional trafficking at the TGN because dysfunction of GARP complex leads to defects in both retrograde trafficking and anterograde trafficking. In murine cells, a complete knockout of all the GARP components resulted in embryonic lethality (Karlsson et al., 2013; Schmitt-John, 2015). Incredulously, a single amino acid substitution in VPS54 subunit resulted in spinal muscular atrophy in the wobbler mouse which is the model for ALS 
(amyotrophic lateral sclerosis). The mutation also leads to a drastic reduction in levels of Vps54, thereby disturbing the GARP complex assembly (Pérez-Victoria et al., 2010). Since cells depleted of either GARP or Golgin seem to display aberrant retrieval of similar cargoes like the MPR or certain toxins, the mechanism of how they select different cargo-enriched carriers is not clear because both tethers interact with the same GTPase Rab6a for proper tethering at the TGN. It is possible that there is some competitive binding involved or there might be some additional factors which select either Golgin or the GARP complex required for tethering. Further exploration is required to understand the mechanism behind the choice of tether.

\section{Fusion at the TGN}

The final step of retrograde transport to the TGN is fusion of the endosomederived transport carrier or vesicle, with the Golgi membrane. Usually, three SNAREs found on a target membrane ( $t$-SNARE) drive fusion by forming a complex with one SNARE found on the vesicle ( $v$-SNARE) (Chen and Scheller, 2001). Until recently, it was widely accepted that the specificity of the SNARE complexes is orchestrated by their inherent ability to bind to each other and form a stable complex (McNew et al., 2000; Parlati et al., 2000), but emerging evidence indicates that some SNAREs tend to be promiscuous. They seem to rely on additional support from membrane tethers to function as stable complexes (Furukawa and Mima, 2014). This concurs with the information provided in the previous section regarding the role of the GARP tether in stabilizing the TGN resident SNARE complex (Abascal-Palacios et al., 2013). SNAREs are structurally characterized by a SNARE motif flanked at one end by a mainly hydrophobic N-terminal 
stretch and at the other end by a C-terminal transmembrane anchor sequence. Most $v$ SNAREs have multiple arginine residues in their SNARE motif, whereas most $t$-SNAREs have numerous glutamine residues in their SNARE motif (Ungermann and Langosch, 2005). Once the transport carrier has been tethered to the Golgi membrane, a 'zippering up event' occurs from the distal N-terminal end to the membrane proximal C-terminal end of the SNAREs to form a stable twisted four-helix bundle, with the SNARE motifs positioned in the midpoint of the bundle. This midpoint is called the zero-layer of the trans-SNARE complex (Ernst and Brunger, 2003). Crystal structure analysis of multiple trans-SNARE complexes reveals that the zero-layer is the site where three glutamine residues from the participating $t$-SNAREs interact with one arginine residue from the corresponding $v$-SNARE (Bock et al., 2001). The formation of the trans-SNARE complex greatly reduces the energy requirement for fusion (Jahn et al., 2003), and helps bring the Golgi and transport carrier membranes close together leading to the merging of lipid bilayers (Dubuke and Munson, 2016; Perez-Victoria and Bonifacino, 2009).

The SNAREs participating in retrograde transport of toxins like $S T x B$ and $C T x B$ are Syntaxin6/Tlg1, Syntaxin16/Tlg2, VAMP3/Snc1, and VTI1a/Vti1 of which VAMP3 is the v-SNARE (Perez-Victoria and Bonifacino, 2009). However, the MPR uses the SNARE Syntaxin10 instead of Syntaxin6 in the same complex mentioned above (F1F) (Zhao et al., 2015). In addition to these, there are two other known SNARE complexes, GS15-Syntaxin5-GS28-Ykt6 and Syntaxin5-Bos1-Bet1-Sec22, which function at the TGN (Furukawa and Mima, 2014; Parlati et al., 2002). The complexity of the SNARE population could be resolved by selectively isolating the subpopulations of SNAREcarrying vesicles, and performing proteomics analysis to understand the relationship 
between SNAREs and corresponding cargo. The diversity of cargo leaving the TEN seems to dictate the need for diversity at every step till it fuses with the TGN.

\section{Problem Statement and Hypothesis}

Cells lacking VPS1 fail to deliver the vacuolar enzyme Carboxypeptidase Y (CPY) from the TGN to the endosome, but rather secretes CPY outside of the cell (Marcusson et al., 1994; Rothman and Stevens, 1986). In addition to CPY mistargeting, the Golgi-resident protein Kex2 is not retrieved from the pre-vacuolar compartments (or late endosome) in VPS1-deficient cells (Nothwehr et al., 1995). Both events of cargo sorting at the TGN and assisting in the retrieval of Golgi proteins from the endosome involve membrane invagination and subsequent pinching-off of emerging vesicles from the donor membrane. It has long been thought that Vps1 serves as a key scission factor to release the invaginated vesicle from different organelles, including the plasma membrane, endosome, and Golgi (Smaczynska-de Rooij et al., 2010). This notion has been supported by the fact that Vps1 shares the sequence homology with mammalian dynamin.

Additionally, a recent discovery by Chi et al. (2014) showed via electron tomography that loss of Vps1 leads to severe accumulation of the CPY receptor Vps10 at the endosomal compartment, which supports the possibility that Vps1 works as a pinchase at the endosome. Nevertheless, the direct evidence that supports that Vps1 pinches off the invaginated vesicle has not been documented. Besides its membrane remodeling role, Vps1 has been considered to play an important role in membrane fusion. In a homotypic vacuolar fusion event, Vps1 binds SNARE Vam3, activates it, and then dissociates from Vam3 to allow the trans-SNARE complex formation (Alpadi et al., 2013; Peters et al., 
2004). Taken together, Vps1 is an emerging factor that plays a role in both membrane scission and fusion. An important, emerging question is whether Vps1 facilitates the tether/fusion event occurring at the TGN. In light of the findings that 1) Vps1 genetically interacts with the TGN tether GARP and that 2) Vps1 localizes to the TGN (Jonikas et al., 2009; Lukehart et al., 2013), I hypothesize that Vps1 functions together with the GARP for vesicle docking necessary for fusion. Several standard molecular biology techniques including yeast two-hybrid assays, cloning, and confocal microscopy were used to address the following four major scientific questions.

1) Does Vps 1 physically interact with one or more of the GARP components? 2) Are there any particular residues implicated in the possible physical interaction?

3) What is the physiological significance of the interaction between Vps1 and the GARP complex?

4) Will Vps1 be an upstream regulator of the GARP complex, or will it work downstream of the complex?

Here I present evidence of physical interaction between Vps1 and Vps51. Mislocalization of Vps51 and Tlg1 in vps $1 \Delta$ cells indicates a role for Vps1 in tether recruitment at the TGN. Finally, the results and discussion sections elucidate the physiological significance of the Vps1-Vps51 interaction. 


\section{MATERIALS AND METHODS}

\section{Plasmid and Yeast Strain Construction}

All yeast strains used in this study are listed in T1, and all bacterial plasmids are listed in T2. Purified genomic DNA (from BY 4741, Invitrogen) was utilized as a template to amplify the required DNA sequences using Phusion Green High-Fidelity DNA Polymerase (Thermo-Fisher Scientific). To tag GFP and mRFP constructs into 3' end of the desired genes, a standard PCR-based gene tagging method (Longtine et al., 1998) was used. To create specific gene deletion strains, such as vps $1 \Delta$ (KKY 0352) and vps51 (KKY 1507), the desired gene was replaced with a KanMX6 and a His3Mx6 module, respectively.

To create p416-TEF-mRFP-Vps1 (KKD 0190), mRFP-Cps1 (KKD 0143) was digested with EcoRI and XhoI to remove CPS1, and then VPS1 sequence was ligated into the same restriction sites of the resulting linear plasmid. To generate pRS426-Gga1mRFP (KKD 0261), the full length sequence of GGA1 and its upstream 200bp sequence, was PCR-amplified and inserted between the NotI and BamHI sites of pRS426 (KKD 0127). The mRFP gene was amplified from mRFP-CPS1 (KKD 0143) and inserted downstream of GGAI into the BamHI and XhoI sites. All insertions were confirmed by colony PCR and restriction digestion. pRS426-Vps51-mRFP (KKD 0298) was made by replacing GGA1 with VPS51 and its upstream 150 bp by using restriction enzymes, NotI and BamHI. KKD 0298 was introduced into a vps $1 \Delta$ strain expressing GFP-Snc1 to create KKY 1697. Construction of the point mutant strains using KKD 0298 as a template is discussed in the section titled 'Site-directed mutagenesis'. The plasmids listed 
above along with plasmids encoding GFP-Snc1 (KKD 0064), GFP-Snc1-PEM (KKD 0062), GFP-Tlg1 (KKD 0069), and Rcy1-GFP (KKD 0067) were introduced via one-step transformation protocol (Chen et al., 1992) into appropriate strains. mRFP-Cps1 was generously donated by Dr. Kihara (Obara et al., 2013) and GFP-Snc1, GFP-Snc1-PEM, Rcy1-GFP, and GFP-Tlg1 were donated by Dr. Fujimura-Kamada (Furuta et al., 2007).

\section{Yeast Two-Hybrid Screening}

All diploid yeast strains utilized for the yeast two-hybrid assay were generated by using the Matchmaker ${ }^{\circledR}$ Gold Yeast Two-Hybrid System (Clontech). Briefly, the PCRamplification of desired sequences was performed by following the standard protocol provided by the In-Fusion ${ }^{\circledR}$ HD Cloning Kit User Manual from Clontech. VPS51 and its fragment sequences were inserted between the $\mathrm{BamHI}$ and $\mathrm{XhoI}$ sites of the prey vector pGADT7 harboring the Activation Domain (AD) (KKD 0083), and the resulting prey vectors are listed in T2. VPS1 and its fragment sequences were inserted into the EcoRI and BamHI sites of the bait vector pGBKT7 harboring the DNA-Binding domain (BD) (KKD 0133) (T2). Insertion of these DNA sequences was confirmed by bacterial colony PCR and restriction digestion. The resulting prey vectors were then transformed into a haploid Y187 strain (KKY 1255) and the bait vector was transformed into the haploid Y2HGOLD strain (KKY 1254). These yeast strains were mated in a flask of 2xYPDA for 24 hours at $30^{\circ} \mathrm{C}$ and, the mated diploid cells were serially diluted by a factor of 3 in a 96 well plate and spotted onto Double Drop Out (DDO) (SD/-Leu/-Trp), Triple Drop Out (TDO) (SD/-Leu/-Trp/-His), and Quadruple Drop Out (QDO) (SD/-Leu/-Trp/-His/-Ade) 
plates. The plates were kept in a stationary incubator at $30^{\circ} \mathrm{C}$ for 3-5 days. Once the cells had grown, images of the plates were captured using the Kodak Image Station 4000MM.

\section{Site-Directed Mutagenesis}

The Glu127 residue of Vps51 was replaced with Lysine (GAA to AAA) using the PhusionTM site-directed mutagenesis kit (Thermo Fisher-Scientific). In summary, purified pGADT7-Vps51 (KKD 0156) or purified pRS426-Vps51-mRFP (KKD 0298) was used a template and amplified with primers carrying the point mutation. Then, the linear DNA product was ligated and introduced into 'Stellar' competent E.coli cells (Clontech). The mutated plasmid DNA was extracted from three colonies using QIAprep ${ }^{\circledR}$ Spin Miniprep Kit (Qiagen), and the presence of the mutation was confirmed by sequencing (Eurofins Genomics). A similar approach was used to create another point mutant Y129F (TAT to TTT) and a double mutant (EY127, 129KF). The mutated copies of Vps51 were introduced into appropriate yeast strains by the transformation protocol mentioned in the previous section.

\section{CMAC Labeling of Yeast Vacuoles}

Yeast vacuoles were selectively stained by incubating the cells with CellTracker ${ }^{\mathrm{TM}}$ CMAC (7-amino4chloromethylcoumarin, Molecular Probes Inc.) as described in Hayden et al. (2013). The stained cells were then examined by conventional microscopy. 


\section{Fluorescence Microscopy}

Fluorescently labeled cells were visualized using an Olympus IX81 microscope equipped with a Yokogawa CSUX1 spinning disk head. The fluorescent images were captured using ImagEM camera (Hamamatsu) for confocal microscopy or Orca camera (Hamamatsu) for conventional microscopy. All strains were grown to mid-log phase (0.60.8 OD), and the images were obtained using a 100X oil immersion objective lens (PlanApo oil objective, numerical aperture 1.4). For simultaneous multi-color imaging, an image splitter (Photometrics ${ }^{\circledR}$ DV2) was used to record both channels at the same time. The exposure was set to $100 \mathrm{~ms}$ for all images.

\section{Statistical Analysis}

The percentage of colocalization between GFP- and mRFP-fused proteins was determined by counting the colocalizing puncta from a minimum of 30 cells, in three individual sets. For the experiments utilizing GFP-Snc1 as a marker, percentage of cells with GFP-Snc1 found at the bud plasma membrane was determined. For all experiments, standard deviation was obtained from the data collected, and a two-tailed T-test was carried out to determine the significance of the experiments performed. Statistical significance was defined as a p-value lesser than 0.05 .

\section{Bioinformatics Tools for Sequence Homology and Homology Modeling}

Sequence homology between ANG2 and Vps51 was identified using a CLUSTALW2 algorithm and the conserved residues are labeled with asterisks. The homology model was generated using SWISS-MODEL tool, and the model was modified 
to indicate the important residues using RasTop software (Glaxo Wellcome Research \& Development). 


\section{RESULTS}

\section{Yeast Two-Hybrid Interaction between Vps1 and Vps51}

A comprehensive genome-wide screen revealing the genetic interaction between VPS1 and all GARP subunits (Jonikas et al., 2009) prompted us to investigate whether one or more GARP subunits physically interact with Vps1. For this, I created four diploid strains expressing each AD-fused GARP subunit (Vps51, Vps52, Vps53 or Vps54) and BD-fused Vps1. A strain co-expressing AD-T and BD-p53 was used as the positive control since p53 interacts with the SV40 large T antigen (DeCaprio et al., 1988). As seen in F2A, only the positive control and the diploid expressing Vps1 and Vps51 grew on TDO and QDO, indicative of a physical interaction between Vps1 and Vps51 in vivo. Vps 1 has three domains, an N-terminal GTPase, a Middle domain, and a C-terminal GTPase Effector Domain (GED) (Moustaq et al., 2016). To identify the domain of Vps1 that binds Vps51, I constructed three strains expressing a BD-fused domain of Vps1 and AD-Vps51. All domains of Vps1 showed high affinity for Vps51 based on the cell growth on both TDO and QDO, implying that multiple sites of Vps1 can bind Vps51 (F2B). To map the region of Vps51 required for interaction with Vps1, several Cterminally truncated variants of Vps51, in increments of 33 amino acids, were fused with AD. These were co-expressed with BD-Vps1, and the resultant strains were grown on DDO, TDO, and QDO. Cell growth was evident on TDO and QDO for the strains expressing the 1-132aa fragment of Vps51 and Vps1. This suggests that the 33aa segment (99-132aa) near the C-terminal end of Vps51 seems to be responsible for the interaction between Vps1 and Vps51 (F2C). When the domains of Vps1 were co-expressed with this 
segment (1-132aa) of Vps51, cell growth was observed just for the N-terminal and Middle domain of Vps1 (F2D), indicating that full length of $\mathrm{Vps} 1$ is required for a strong interaction with Vps51. Together, the results imply that a 33aa segment of Vps51 interacts with the GTPase and Middle domain of Vps1 in vivo.

\section{Two Residues, E127 and Y129 of Vps51 are Implicated in its Interaction with Vps1}

To more precisely characterize the interaction between Vps1 and Vps51, I aligned the 33aa stretch (99-132aa) of Vps51 with its mammalian homolog Ang2. From the alignment results (F2E), I identified six residues which were $100 \%$ conserved. Among those six, two were sporadic, and the remaining four residues (YENY, 126-129aa) were found in a row. To examine whether this 4 amino acid stretch is responsible for Vps 1 interaction, I created three AD-fused Vps51 mutant variants, Vps51 ${ }^{\mathrm{E} 127 \mathrm{~K}}$, Vps $51^{\mathrm{Y} 129 \mathrm{~F}}$, and Vps51 ${ }^{\mathrm{EY} 127,129 \mathrm{KF}}$. These fusion proteins were co-expressed with BD-Vps1 in diploid cells, which were spotted onto DDO, TDO, and QDO. Cell growth was seen only from cells co-expressing full length Vps1 and Vps51, but not from cells harboring any of the Vps51 mutants, indicating that the mutant versions of Vps51 do not interact with Vps1 in vivo $(\mathrm{F} 2 \mathrm{~F})$.

To exclude the possibility that those substitution mutations affect cell growth, I performed a colony growth assay wherein all the mutant strains were grown on suitable media. The mutant strains grew at a rate similar to wild-type (F2G), suggesting that cell growth remained unaffected by the expression of the Vps51 mutants and that the lack of growth on TDO and QDO was simply due to no interaction between Vps1 and Vps51 mutants. 


\section{Vps51 Colocalizes with Vps1 and the Loss of Vps1 Affects Localization of Vps51 and TIg1 at the Late Golgi}

Previous studies revealed that Vps1 localizes to the TGN (Lukehart et al., 2013). In light of the finding that Vps1 and Vps51 physically interact (F2), I investigated the possibility of Vps51 colocalizing with Vps1. For this, Vps1 was tagged at its N-terminal with mRFP, and Vps51 was tagged at its C-terminal with GFP. These tagged constructs were co-expressed in a wild-type strain, and the extent of colocalization between them was determined using data collected by confocal microscopy. It was found that $22 \% \pm$ 1.21 of mRFP-Vps1 puncta colocalized with Vps51-GFP puncta (F3A). To test whether Vps1 is required for Vps51 recruitment to the Golgi, I constructed a strain expressing Vps51-GFP and the late Golgi marker Gga1-mRFP (Ghosh and Kornfeld, 2004). As seen in $\mathrm{F} 3 \mathrm{~B}, 64 \% \pm 0.88$ of Gga1-mRFP puncta colocalized with Vps51-GFP puncta in wildtype cells. However, only $29.9 \% \pm 2.01$ of Ggal-mRFP puncta colocalized with Vps51GFP in a $v p s 1 \Delta$ background $(\mathrm{p}<0.001)$, implying that $\mathrm{Vps} 1$ is required for proper recruitment of Vps51 to the Golgi.

Vps51 interacts with the late Golgi SNARE Tlg1 for efficient transport carrier fusion at the TGN (Siniossoglou and Pelham, 2002b). Given the severe mistargeting of Vps51 in the absence of Vps1, I reasoned the possibility that Tlg1's Golgi localization would also be disturbed in vps $1 \Delta$ cells. Quantitation of colocalization levels of Sec7-RFP, a late Golgi marker (McDonold and Fromme, 2014) with GFP-Tlg1 showed that $50.5 \% \pm$ 1.11 of Sec7-RFP dots coincide with GFP-Tlg1 puncta in wild type cells. In contrast, $v p s 1 \Delta$ cells display only $11.9 \% \pm 0.35$ of Sec 7 -RFP puncta colocalization with GFP-Tlg1 puncta $(\mathrm{p}<0.001)$. Most Tlg1 puncta appeared to accumulate on the rim of the vacuole 
instead of the TGN. The cells were stained with CellTrackerTM CMAC to mark the vacuolar lumen and to affirm mislocalization of Tlg1 to the rim of the vacuole (F3C).

\section{The Functional Relationship between Vps1 and Vps51 during Tethering/Fusion at the TGN}

Snc1, a recycling SNARE protein at the plasma membrane, is internalized toward the early endosome and then delivered to the TGN (Gerst et al., 1992). Loss of either Vps1 or Vps51 result in an aberrant phenotype of Snc1 (Burston et al., 2009; Palmer et al.). In correlation with this, the vast majority $(83.3 \% \pm 3.33)$ of wild type cells expressing GFP-Snc1 were polarized at the bud, when compared to $23.3 \% \pm 10$ of $v p s 1 \Delta$ cells $(\mathrm{p}<0.001)$ and $31.1 \% \pm 1.92$ of vps $51 \Delta$ cells $(\mathrm{p}<0.001)(\mathrm{F} 4 \mathrm{~A} \& \mathrm{~B})$. Importantly, a majority of the vps $1 \Delta$ and $v p s 51 \Delta$ cells displayed internal puncta of GFP-Snc1 (F4A). The identity of this cytoplasmic puncta is described in detail in the discussion section. By utilizing an endocytosis-defective variant of Snc1, Snc1-PEM, I observed that the mutant Snc1 is found at the plasma membrane in all strains used in F4A \& B, indicating that it is secreted normally from the Golgi to the membrane (F4C). Consistent with published evidence, my results confirmed that Vps1 and Vps51 work in the retrograde pathway from endosome-to-TGN, and absence of either Vps1 or Vps51 leads to dysfunctional tethering/fusion of Snc1-laden transport carriers.

Next, I proceeded to clarify the functional relationship between Vps1 and Vps51 during tethering/fusion. In the absence of an upstream effector, overexpression of a downstream factor might rescue the process (Kofron et al., 1999). Thus, if Vps1 works upstream of Vps51, the percentage of cells displaying polarized Snc1 must be higher 
when Vps51 is overexpressed in vps $1 \Delta$ cells and not the other way around. In support of my notion, the results indicate that $46 \% \pm 5.29$ of $v p s 1 \Delta$ cells with overexpression of Vps51 displayed GFP-Snc1 polarization when compared to $14.33 \% \pm 2.08$ of $v p s 1 \Delta$ cells $(\mathrm{p}<0.001)$. On the other hand, $24 \% \pm 1.32$ of $v p s 51 \Delta$ cells with overexpression of Vps 1 displayed GFP-Snc1 polarization when compared to $30 \% \pm 1.56$ of $v p s 51 \Delta$ cells, providing evidence that Vps1 works upstream of Vps51 during tethering/fusion (F4D \& E).

\section{Physiological Significance of Vps1-Vps51 Interaction}

To assess the physiological significance of the physical interaction of Vps1 with Vps51 in the context of endosome-to-TGN traffic, the full length of Vps51 or Vps51 mutants (Vps51 ${ }^{\mathrm{E} 127 \mathrm{~K}}$, Vps51 ${ }^{\mathrm{Y} 129 \mathrm{~F}}$, or Vps51 ${ }^{\mathrm{EY} 127,129 \mathrm{KF}}$ ) were co-expressed with GFPSnc1 in vps $51 \Delta$ cells. If the interaction of Vps51 with Vps1 via these conserved residues is physiologically relevant, then in the presence of any of the Vps51 mutants there should be a significant decrease in Snc1 polarization. Correspondingly, $68.8 \% \pm 1.92$ of cells expressing full length of Vps51 showed polarized Snc1 when compared to $50 \% \pm 3.33$ of cells expressing Vps51 ${ }^{\mathrm{E} 127 \mathrm{~K}}(\mathrm{p}<0.001)$ and $58.8 \% \pm 3.84$ of cells expressing Vps51 ${ }^{\mathrm{Y} 129 \mathrm{~F}}$ $(\mathrm{p}<0.01)$. When the double mutant copy of Vps51 (Vps51 $\left.{ }^{\mathrm{EY} 127,129 \mathrm{KF}}\right)$ was expressed, $53.3 \% \pm 6.66$ of cells exhibited GFP-Snc1 polarization ( $<<0.01)$ (F5). Due to the statistically significant difference in the rescue of Snc1 phenotype between a normal copy of Vps51 and a mutant copy of Vps51, it appears that disruption of the interaction between Vps1 and Vps51 results in improper tethering, thereby faulty fusion. 
However, the interaction of Vps1 and Vps51 via these specific residues doesn't seem to be necessary for the recruitment of Vps51 to the TGN (F6A). It was observed that $55 \% \pm 5.45$ of Vps51-mRFP puncta, $56 \% \pm 2.39$ of Vps51 ${ }^{\text {E127K }}$-mRFP puncta, $58 \%$ \pm 4.6 of $\mathrm{Vps} 51^{\mathrm{Y} 129 \mathrm{~F}}-\mathrm{mRFP}$ puncta, and $53 \% \pm 3.66$ of $\mathrm{Vps} 51^{\mathrm{EY} 127,129 \mathrm{KF}}$-mRFP puncta colocalized with late Golgi marker Rcyl-GFP (Lukehart et al., 2013). The expression of point mutants or full length Vps51 did not make a statistically significant difference in the percentage of colocalization of Vps51 with Rcyl-GFP. 


\section{DISCUSSION}

Findings from my study have identified a novel biochemical mechanism of interaction between Vps1 and Vps51, a GARP component. The physical interaction has a physiological relevance in the context of heterotypic fusion of endosome-derived vesicles at the TGN. Furthermore, my results point toward Vps1 working as part of a local protein-network responsible for tethering/fusion at the TGN.

Retrograde transport from the endosomes to the TGN was first discovered when the MPR recycled to the TGN, after transporting acid hydrolases to the endosome (Griffiths et al., 1988). Vps10, which also transports acid hydrolases in yeast, follows the retrograde pathway to be retrieved to the TGN (Marcusson et al., 1994). Since then this pathway has been extensively studied in both mammals and yeast, and as a result many different cargoes including toxins, viruses, and SNARE proteins that are retrieved by this pathway, have been identified. Snc1, a yeast SNARE, has been used as a prominent marker for delineating retrograde transport from the endosomes to the TGN (Protopopov et al., 1993; Robinson et al., 2006). Snc1 is synthesized in the ER, transported to the TGN, and secreted to the plasma membrane. Then, it is endocytosed, trafficked to the early endosome, and finally delivered to the TGN (Lewis et al., 2000b). The transport carrier with the cargo Snc1 is peripherally associated with Ypt6, a small GTPase, which interacts with Vps52, a GARP subunit, to facilitate recognition of the carrier at the TGN (Chia and Gleeson, 2011). Another GARP subunit Vps51 interacts with Tlg1, a $t$-SNARE at the TGN, connecting the transport carrier to the TGN (Siniossoglou and Pelham, 2002b). This Ypt6-Vps52-Vps51-Tlg1 network/interaction secures the GARP tether to 
efficiently anchor the transport carrier at the TGN. After tethering, the trans-SNARE complex consisting of Tlg1-Tlg2-Vti1-Snc1 is formed, culminating in the fusion of the endosome-derived transport carrier with the TGN (Parlati et al., 2002).

The observation of accumulated cytoplasmic GFP-Snc1 puncta in $v p s 1 \Delta$ cells (F4A) is consistent with previously published data (Lukehart et al., 2013; Smaczynska-de Rooij et al., 2012). It is most likely that the cytoplasmic Snc1-carrying compartments might be early endosomes because the puncta are exclusively internal (F4A), indicating the endocytic process is not significantly hampered in the vps 1 mutant cells. Additionally, an endocytosis-deficient mutant of Snc1 is properly targeted to the plasma membrane in vps $1 \Delta$ cells, reflecting no defects in the secretory pathway (F4C), which reaffirms the idea that Snc1 puncta are early endosomes. Nevertheless, the precise physiological role of Vps1 in endosome-to-TGN traffic of Snc1-laden transport carriers has not been documented. One of the plausible explanations for the observed abnormal phenotype of Snc1 would be that the pinching-off process of Snc1-laden tubules at the endosome is defective, in the case that Vps1 serves as a pinchase at the endosome. It is well known that Vps10-laden tubules accumulate at the endosome and that individual tubules appear to be elongated in $v p s 1 \Delta$ cells, supporting the role of Vps1 as a pinchase at the endosome (Chi et al., 2014). However, it has been proposed that Vps10 is trafficked from the late endosome to the TGN, and hence there is a limitation on extrapolating the role of Vps1 as a pinchase to Snc1-laden tubules, because it is trafficked from the early endosome to the TGN (Lewis et al., 2000a; Pfeffer, 2011). The question of a universal role for Vps1 in fission of early or late endosome tubules awaits further exploration. An alternative explanation for the Snc1 distribution defect would be improper tethering/fusion 
preventing the Snc1-laden transport carrier from fusing with the TGN. In order for this to be plausible, Vps1 must function as a part of tethering/fusion machinery. For any protein to be involved in a specific process, it has to be physically/functionally associated with the machinery that coordinates the process. Results from my two-hybrid experiments revealed the physical interaction of Vps51 with Vps1 via the 33 amino acid segment (99132) near the C-terminal of Vps51 (F2A-D). Therefore, it is reasonable to postulate that Vps1 is a part of the Ypt6-Vps52-Vps51-Tlg1 network mentioned earlier, and the functional relationship of $\mathrm{Vps} 1$ with the components of this network is discussed in the following section.

Sometimes, overexpression of a downstream factor in the absence of an upstream regulator can rescue the process mediated by these factors (Li et al., 2000). Partial rescue of the aberrant phenotype of GFP-Snc1 was seen when Vps51 was overexpressed in vps $1 \Delta$ cells, but not vice versa, suggesting the possibility that $\mathrm{Vps} 1$ is an upstream regulator of Vps51 (F4D,E \& 7). However, one cannot exclude the possibility that in the absence of Vps1, the excess amount of Vps51 interacts in a stable manner with Tlg1 or another unknown participant of tethering/fusion event, thus orchestrating favorable conditions for the process. What is the role of Vps1 in this multi-protein network needed for tethering/fusion? It appears that Vps1 plays an important role in recruiting Vps51 to the TGN in light of the finding that the extent of colocalization of Vps51 with a late Golgi marker was severely reduced, albeit not completely, in vps $1 \Delta$ cells (F3B). Yet, the exact subcellular location that Vps51 is mistargeted to remains unidentified and requires further research. The rationale for the minor fraction of Vps51 that is properly targeted to the Golgi in vps $1 \Delta$ cells is that Vps51 could be recruited by Ypt6 as it has been 
implicated in the recruitment of Vps51 (Rosa-Ferreira et al., 2015; Siniossoglou and Pelham, 2002b). This suggests that Vps1 and Ypt6 might work in parallel or in collaboration, to recruit Vps51 to the TGN. As mentioned earlier, the last component of this functional network is Tlg1. Given that a large fraction of Tlg1 appeared to be mistargeted to the vacuolar rim in $v p s 1 \Delta$ cells $(\mathrm{F} 3 \mathrm{C})$, one can conjecture that the SNARE complex between the TGN and the endosome-derived transport carrier is not formed properly due to the inherent specificity of SNARE complexes (Chen and Scheller, 2001). Yet this novel observation is surprising because prior results from my laboratory have shown that Tlg1 does not interact genetically (Lukehart et al., 2013) or physically with Vps1 (unpublished data). Vps51 directly interacts with Tlg1 (Siniossoglou and Pelham, 2002a), leading one to surmise that the mistargeting of Tlg1 in vps $1 \Delta$ cells is likely a result of a negative domino effect, wherein Vps51's mislocalization leads to mislocalization of its downstream factor. It is well known that loss of Vps 1 also results in mistargeting of Vps10 and Kex2 to the vacuole (Brummett et al., 2013). Therefore, a more likely possibility is that there is a global retrieval defect in $v p s 1 \Delta$ cells because mistargeting of Tlg1 phenocopies the mistargeting of Vps10 and Kex2. Taken together, it seems that the supramolecular complex needed for tethering/fusion doesn't assemble properly if Vps1 is absent, validating the inclusion of Vps1 in the existing network for tethering/fusion, Ypt6-GARP-SNARE (Conibear et al., 2003) (F7). The relationship between Vps1 and Ypt6 is not entirely clear and, further research on the relationship is required to complete this interaction cascade.

The present study revealed that two conserved residues, E127 and Y129, of Vps51 directly interact with Vps1 (F2E \& F). A group of researchers previously proposed 
that these residues are situated in a coiled-coil domain (73-164aa) near the C-terminal of Vps51 (Siniossoglou and Pelham, 2002a). According to a homology model I constructed using SWISSMODEL and RasTop, the residues E127 and Y129 are exposed to the solvent (F6B). A possible reasoning for the loss of interaction between Vps1 and the mutant Vps51 harboring mutations in those residues could be that the creation of point mutations in this region of Vps51 affects negatively the integrity of the coiled coil, thereby abolishing interaction with Vps1.

The physiological significance of this interaction was studied by exogenously expressing Snc1 in vps51 $1 \Delta$ cells wherein the sole copy of Vps51 was either the point mutants of the residues mentioned previously or the full length of Vps51. Both the point mutants, Vps51 ${ }^{\mathrm{E} 127 \mathrm{~K}}$ and $\mathrm{Vps} 51^{\mathrm{Y} 129 \mathrm{~F}}$, were not able to rescue the aberrant phenotype of Snc1 with the same efficiency as the full length of Vps51 was overexpressed (F4G), strongly suggesting that disturbing the interaction between Vps1 and Vps51 possibly results in a tethering defect that might lead to improper fusion. Furthermore, point mutations of these residues didn't show any significant changes in the localization of Vps51, suggesting that interaction between Vps1 and these residues in Vps51 is not crucial for recruitment of Vps51 (F6A \& 7). This results signify that the interaction of Vps1 with these residues in Vps51 is exclusively implicated in modulating the tethering activity of the GARP. One possible explanation for proper targeting of the Vps51 mutant species to the late Golgi is that intact Ypt6 might recruit the mutant Vps51 species that present the binding region for Ypt6. Alternatively, it may be that Vps51 possess more Vps1 binding sites that have not been detected due to a feeble transient interaction with Vps1 in the yeast two hybrid system I used. Further research is required to properly 
address either possibility. Although the Vps51 mutants localize at the TGN, their role as an efficient tether still remains compromised because there is a tethering/fusion defect when the point mutants are expressed. Considering my results, I propose a model providing a mechanistic description of tethering/fusion at the TGN in wild type and vps $1 \Delta$ backgrounds (F7). The model depicts the significance of Vps1 functioning as an important factor of a local protein network consisting of GARP and SNAREs at the contact point of the endosome-derived transport carrier and the TGN, which is required for efficient tethering/fusion at the TGN. 


\section{REFERENCES}

Abascal-Palacios, G., Schindler, C., Rojas, A.L., Bonifacino, J.S., and Hierro, A. (2013). Structural basis for the interaction of the golgi-associated retrograde protein complex with the t-SNARE syntaxin 6. Structure 21, 1698-1706.

Ackema, K.B., Sauder, U., Solinger, J.A., and Spang, A. (2013). The ArfGEF GBF-1 is required for ER structure, secretion and endocytic transport in C. elegans. PloS one 8 , e67076.

Alpadi, K., Kulkarni, A., Namjoshi, S., Srinivasan, S., Sippel, K.H., Ayscough, K., Zieger, M., Schmidt, A., Mayer, A., Evangelista, M., et al. (2013). DynaminSNARE interactions control trans-SNARE formation in intracellular membrane fusion. Nat Commun 4, 1704.

Anitei, M., Chenna, R., Czupalla, C., Esner, M., Christ, S., Lenhard, S., Korn, K., Meyenhofer, F., Bickle, M., and Zerial, M. (2014). A high-throughput siRNA screen identifies genes that regulate mannose 6-phosphate receptor trafficking. J Cell Sci 127, 5079-5092.

Antonny, B., Burd, C., De Camilli, P., Chen, E., Daumke, O., Faelber, K., Ford, M., Frolov, V.A., Frost, A., and Hinshaw, J.E. (2016). Membrane fission by dynamin: what we know and what we need to know. The EMBO Journal, e201694613.

Atencia, J., and Beebe, D.J. (2005). Controlled microfluidic interfaces. Nature 437, 648655.

Balla, T. (2016). Cell biology: Lipid code for membrane recycling. Nature 529, 292-293.

Belyy, V., Schlager, M.A., Foster, H., Reimer, A.E., Carter, A.P., and Yildiz, A. (2016a). The mammalian dynein-dynactin complex is a strong opponent to kinesin in a tug-of-war competition. Nat Cell Biol 18, 1018-1024.

Belyy, V., Schlager, M.A., Foster, H., Reimer, A.E., Carter, A.P., and Yildiz, A. (2016b). The mammalian dynein-dynactin complex is a strong opponent to kinesin in a tug-of-war competition. Nature Cell Biology 18, 1018-1024.

Besterman, J.M., and Low, R.B. (1983). Endocytosis: a review of mechanisms and plasma membrane dynamics. Biochemical Journal 210, 1.

Bock, J.B., Matern, H.T., Peden, A.A., and Scheller, R.H. (2001). A genomic perspective on membrane compartment organization. Nature 409, 839-841.

Bohley, P. (1995). The fates of proteins in cells. Naturwissenschaften 82, 544-550. 
Bonifacino, J.S., and Dell'Angelica, E.C. (1999). Molecular bases for the recognition of tyrosine-based sorting signals. The Journal of cell biology 145, 923-926.

Bonifacino, J.S., and Rojas, R. (2006a). Retrograde transport from endosomes to the trans-Golgi network. Nature Reviews Molecular Cell Biology 7, 568-579.

Bonifacino, J.S., and Rojas, R. (2006b). Retrograde transport from endosomes to the trans-Golgi network. Nature reviews Molecular cell biology 7, 568-579.

Borner, G.H., Antrobus, R., Hirst, J., Bhumbra, G.S., Kozik, P., Jackson, L.P., Sahlender, D.A., and Robinson, M.S. (2012). Multivariate proteomic profiling identifies novel accessory proteins of coated vesicles. J Cell Biol 197, 141-160.

Brummett, A., Williams, M., Hayden, J., and Kim, K. (2013). Implication of Yeast Dynamin-Related Protein Vps1 in Endocytosis and Organelle Fission. International Journal of Science and Technology 2.

Burd, C.G. (2011). Physiology and pathology of endosome-to-Golgi retrograde sorting. Traffic 12, 948-955.

Chen, Y.A., and Scheller, R.H. (2001). SNARE-mediated membrane fusion. Nature reviews Molecular cell biology 2, 98-106.

Cheung, P.Y., and Pfeffer, S.R. (2016). Transport Vesicle Tethering at the Trans Golgi Network: Coiled Coil Proteins in Action. Front Cell Dev Biol 4, 18.

Chi, R.J., Liu, J., West, M., Wang, J., Odorizzi, G., and Burd, C.G. (2014). Fission of SNX-BAR-coated endosomal retrograde transport carriers is promoted by the dynamin-related protein Vps1. The Journal of cell biology 204, 793-806.

Chia, P.Z.C., and Gleeson, P.A. (2011). The Regulation of Endosome-to-Golgi Retrograde Transport by Tethers and Scaffolds. Traffic (Copenhagen, Denmark) $12,939-947$.

Collins, B.M. (2008). The structure and function of the retromer protein complex. Traffic 9, 1811-1822.

Conibear, E., Cleck, J.N., and Stevens, T.H. (2003). Vps51p mediates the association of the GARP (Vps52/53/54) complex with the late Golgi t-SNARE Tlg1p. Molecular biology of the cell 14, 1610-1623.

Curtiss, M., Jones, C., and Babst, M. (2007). Efficient cargo sorting by ESCRT-I and the subsequent release of ESCRT-I from multivesicular bodies requires the subunit Mvb12. Molecular biology of the cell 18, 636-645.

Damke, H., Binns, D.D., Ueda, H., Schmid, S.L., and Baba, T. (2001). Dynamin GTPase domain mutants block endocytic vesicle formation at morphologically distinct stages. Molecular biology of the cell 12, 2578-2589. 
de Boer, P., Hoogenboom, J.P., and Giepmans, B.N. (2015). Correlated light and electron microscopy: ultrastructure lights up! Nature methods 12, 503-513.

Derby, M.C., Lieu, Z.Z., Brown, D., Stow, J.L., Goud, B., and Gleeson, P.A. (2007). The trans-Golgi network golgin, GCC185, is required for endosome-to-Golgi transport and maintenance of Golgi structure. Traffic 8, 758-773.

Derivery, E., Sousa, C., Gautier, J.J., Lombard, B., Loew, D., and Gautreau, A. (2009). The Arp2/3 activator WASH controls the fission of endosomes through a large multiprotein complex. Dev Cell 17, 712-723.

Du Toit, A. (2015). Endocytosis: A new gateway into cells. Nat Rev Mol Cell Biol 16, 68-68.

Dubuke, M.L., and Munson, M. (2016). The Secret Life of Tethers: The Role of Tethering Factors in SNARE Complex Regulation. Front Cell Dev Biol 4, 42.

Efimov, A., Kharitonov, A., Efimova, N., Loncarek, J., Miller, P.M., Andreyeva, N., Gleeson, P., Galjart, N., Maia, A.R., McLeod, I.X., et al. (2007). Asymmetric CLASP-dependent nucleation of noncentrosomal microtubules at the trans-Golgi network. Dev Cell 12, 917-930.

Ernst, J.A., and Brunger, A.T. (2003). High resolution structure, stability, and synaptotagmin binding of a truncated neuronal SNARE complex. Journal of Biological Chemistry 278, 8630-8636.

Ferguson, S.M., and De Camilli, P. (2012). Dynamin, a membrane-remodelling GTPase. Nature reviews Molecular cell biology 13, 75-88.

Follett, J., Bugarcic, A., Yang, Z., Ariotti, N., Norwood, S.J., Collins, B.M., Parton, R.G., and Teasdale, R.D. (2016). Parkinson disease-linked Vps35 R524W mutation impairs the endosomal association of retromer and induces $\alpha$-synuclein aggregation. Journal of Biological Chemistry 291, 18283-18298.

Fridmann-Sirkis, Y., Kent, H.M., Lewis, M.J., Evans, P.R., and Pelham, H.R. (2006). Structural analysis of the interaction between the SNARE Tlg1 and Vps51. Traffic 7, 182-190.

Fridmann-Sirkis, Y., Siniossoglou, S., and Pelham, H.R. (2004). TMF is a golgin that binds Rab6 and influences Golgi morphology. BMC Cell Biol 5, 18.

Furukawa, N., and Mima, J. (2014). Multiple and distinct strategies of yeast SNAREs to confer the specificity of membrane fusion. Scientific reports 4 .

Furuta, N., Fujimura-Kamada, K., Saito, K., Yamamoto, T., and Tanaka, K. (2007). Endocytic recycling in yeast is regulated by putative phospholipid translocases and the Ypt31p/32p-Rcylp pathway. Mol Biol Cell 18, 295-312. 
Galiani, S., Waithe, D., Reglinski, K., Cruz-Zaragoza, L.D., Garcia, E., Clausen, M.P., Schliebs, W., Erdmann, R., and Eggeling, C. (2016). Super-resolution Microscopy Reveals Compartmentalization of Peroxisomal Membrane Proteins. Journal of Biological Chemistry 291, 16948-16962.

Gautreau, A., Oguievetskaia, K., and Ungermann, C. (2014). Function and regulation of the endosomal fusion and fission machineries. Cold Spring Harbor perspectives in biology 6 , a016832.

Gershlick, D.C., Schindler, C., Chen, Y., and Bonifacino, J.S. (2016). TSSC1 is novel component of the endosomal retrieval machinery. Molecular biology of the cell 27, 2867-2878.

Griffiths, G., Hoflack, B., Simons, K., Mellman, I., and Kornfeld, S. (1988). The mannose 6-phosphate receptor and the biogenesis of lysosomes. Cell 52, 329-341.

Hao, M., and Maxfield, F.R. (2000). Characterization of rapid membrane internalization and recycling. Journal of Biological Chemistry 275, 15279-15286.

Harbour, M.E., Breusegem, S.Y., and Seaman, M.N. (2012). Recruitment of the endosomal WASH complex is mediated by the extended 'tail' of Fam 21 binding to the retromer protein Vps35. Biochem J 442, 209-220.

Harrison, M.S., Hung, C.S., Liu, T.T., Christiano, R., Walther, T.C., and Burd, C.G. (2014). A mechanism for retromer endosomal coat complex assembly with cargo. Proc Natl Acad Sci U S A 111, 267-272.

Hayden, J., Williams, M., Granich, A., Ahn, H., Tenay, B., Lukehart, J., Highfill, C., Dobard, S., and Kim, K. (2013). Vps1 in the late endosome-to-vacuole traffic. Journal of Biosciences 38, 73-83.

Hayes, G.L., Brown, F.C., Haas, A.K., Nottingham, R.M., Barr, F.A., and Pfeffer, S.R. (2009). Multiple Rab GTPase binding sites in GCC185 suggest a model for vesicle tethering at the trans-Golgi. Molecular biology of the cell 20, 209-217.

Hong, Z., Yang, Y., Zhang, C., Niu, Y., Li, K., Zhao, X., and Liu, J.J. (2009). The retromer component SNX6 interacts with dynactin p150(Glued) and mediates endosome-to-TGN transport. Cell research 19, 1334-1349.

Huang, M., Bai, Y., Sjostrom, S.L., Hallström, B.M., Liu, Z., Petranovic, D., Uhlén, M., Joensson, H.N., Andersson-Svahn, H., and Nielsen, J. (2015). Microfluidic screening and whole-genome sequencing identifies mutations associated with improved protein secretion by yeast. Proceedings of the National Academy of Sciences 112, E4689-E4696.

Jahn, R., Lang, T., and Südhof, T.C. (2003). Membrane Fusion. Cell 112, 519-533. 
Jonikas, M.C., Collins, S.R., Denic, V., Oh, E., Quan, E.M., Schmid, V., Weibezahn, J., Schwappach, B., Walter, P., Weissman, J.S., et al. (2009). Comprehensive characterization of genes required for protein folding in the endoplasmic reticulum. Science 323, 1693-1697.

Kamagata, E., Kudo, T., Kimura, R., Tanimukai, H., Morihara, T., Sadik, M.G., Kamino, K., and Takeda, M. (2009). Decrease of dynamin 2 levels in late-onset Alzheimer's disease alters $A \beta$ metabolism. Biochemical and biophysical research communications 379, 691-695.

Karlsson, P., Droce, A., Moser, J.M., Cuhlmann, S., Padilla, C.O., Heimann, P., Bartsch, J.W., Füchtbauer, A., Füchtbauer, E.-M., and Schmitt-John, T. (2013). Loss of vps54 function leads to vesicle traffic impairment, protein mis-sorting and embryonic lethality. International journal of molecular sciences 14, 10908-10925.

Kollmann, K., Pohl, S., Marschner, K., Encarnação, M., Sakwa, I., Tiede, S., Poorthuis, B.J., Lübke, T., Müller-Loennies, S., and Storch, S. (2010). Mannose phosphorylation in health and disease. European journal of cell biology $89,117-$ 123.

Kornfeld, S. (1992). Structure and function of the mannose 6-phosphate/insulinlike growth factor II receptors. Annual review of biochemistry 61, 307-330.

Kühling, L., and Schelhaas, M. (2014). Systematic analysis of endocytosis by cellular perturbations. Exocytosis and Endocytosis, 19-46.

Lauvrak, S.U., Torgersen, M.L., and Sandvig, K. (2004). Efficient endosome-to-Golgi transport of Shiga toxin is dependent on dynamin and clathrin. J Cell Sci 117, 2321-2331.

Lee, I., Doray, B., Govero, J., and Kornfeld, S. (2008). Binding of cargo sorting signals to AP-1 enhances its association with ADP ribosylation factor 1-GTP. The Journal of cell biology 180, 467-472.

Lewis, M.J., Nichols, B.J., Prescianotto-Baschong, C., Riezman, H., and Pelham, H.R. (2000a). Specific retrieval of the exocytic SNARE Snclp from early yeast endosomes. Molecular biology of the cell 11,23-38.

Lewis, M.J., Nichols, B.J., Prescianotto-Baschong, C., Riezman, H., and Pelham, H.R. (2000b). Specific retrieval of the exocytic SNARE Snc1p from early yeast endosomes. Molecular biology of the cell 11,23-38.

Li, P.-X., Wong, J., Ayed, A., Ngo, D., Brade, A.M., Arrowsmith, C., Austin, R.C., and Klamut, H.J. (2000). Placental transforming growth factor- $\beta$ is a downstream mediator of the growth arrest and apoptotic response of tumor cells to DNA damage and p53 overexpression. Journal of Biological Chemistry 275, 2012720135. 
Llorente, A., Rapak, A., Schmid, S.L., Van Deurs, B., and Sandvig, K. (1998). Expression of mutant dynamin inhibits toxicity and transport of endocytosed ricin to the Golgi apparatus. The Journal of cell biology 140, 553-563.

Longtine, M.S., McKenzie, A., 3rd, Demarini, D.J., Shah, N.G., Wach, A., Brachat, A., Philippsen, P., and Pringle, J.R. (1998). Additional modules for versatile and economical PCR-based gene deletion and modification in Saccharomyces cerevisiae. Yeast (Chichester, England) 14, 953-961.

Lukehart, J., Highfill, C., and Kim, K. (2013). Vps1, a recycling factor for the traffic from early endosome to the late Golgi. Biochemistry and cell biology $=$ Biochimie et biologie cellulaire 91, 455-465.

Mallard, F., Antony, C., Tenza, D., Salamero, J., Goud, B., and Johannes, L. (1998). Direct pathway from early/recycling endosomes to the Golgi apparatus revealed through the study of shiga toxin B-fragment transport. The Journal of cell biology 143, 973-990.

Marcusson, E.G., Horazdovsky, B.F., Cereghino, J.L., Gharakhanian, E., and Emr, S.D. (1994). The sorting receptor for yeast vacuolar carboxypeptidase $Y$ is encoded by the VPS10 gene. Cell 77, 579-586.

Matsudaira, T., Niki, T., Taguchi, T., and Arai, H. (2015). Transport of the cholera toxin B-subunit from recycling endosomes to the Golgi requires clathrin and AP-1. J Cell Sci 128, 3131-3142.

McGough, I.J., and Cullen, P.J. (2011). Recent advances in retromer biology. Traffic 12, 963-971.

McGough, I.J., and Cullen, P.J. (2013a). Clathrin is not required for SNX-BAR-retromermediated carrier formation. Journal of Cell Science 126, 45-52.

McGough, I.J., and Cullen, P.J. (2013b). Clathrin is not required for SNX-BAR-retromermediated carrier formation. J Cell Sci 126, 45-52.

McNew, J.A., Parlati, F., Fukuda, R., Johnston, R.J., Paz, K., Paumet, F., Sollner, T.H., and Rothman, J.E. (2000). Compartmental specificity of cellular membrane fusion encoded in SNARE proteins. Nature 407, 153-159.

Mesaki, K., Tanabe, K., Obayashi, M., Oe, N., and Takei, K. (2011). Fission of tubular endosomes triggers endosomal acidification and movement. PloS one 6, e19764.

Miller, P.M., Folkmann, A.W., Maia, A.R., Efimova, N., Efimov, A., and Kaverina, I. (2009). Golgi-derived CLASP-dependent microtubules control Golgi organization and polarized trafficking in motile cells. Nature cell biology 11, 1069-1080.

Moore, M.S., Mahaffey, D.T., Brodsky, F.M., and Anderson, R. (1987). Assembly of clathrin-coated pits onto purified plasma membranes. Science 236, 558-563. 
Muhammad, A., Flores, I., Zhang, H., Yu, R., Staniszewski, A., Planel, E., Herman, M., Ho, L., Kreber, R., and Honig, L.S. (2008). Retromer deficiency observed in Alzheimer's disease causes hippocampal dysfunction, neurodegeneration, and $\mathrm{A} \beta$ accumulation. Proceedings of the National Academy of Sciences 105, 7327-7332.

Nicoziani, P., Vilhardt, F., Llorente, A., Hilout, L., Courtoy, P.J., Sandvig, K., and van Deurs, B. (2000). Role for dynamin in late endosome dynamics and trafficking of the cation-independent mannose 6-phosphate receptor. Molecular biology of the cell 11, 481-495.

Nisar, S., Kelly, E., Cullen, P.J., and Mundell, S.J. (2010). Regulation of P2Y1 Receptor Traffic by Sorting Nexin 1 is Retromer Independent. Traffic 11, 508-519.

Nothwehr, S.F., Conibear, E., and Stevens, T.H. (1995). Golgi and vacuolar membrane proteins reach the vacuole in vps 1 mutant yeast cells via the plasma membrane. The Journal of Cell Biology 129, 35-46.

Obara, K., Kojima, R., and Kihara, A. (2013). Effects on vesicular transport pathways at the late endosome in cells with limited very long-chain fatty acids. J Lipid Res 54, 831-842.

Parlati, F., McNew, J.A., Fukuda, R., Miller, R., Sollner, T.H., and Rothman, J.E. (2000). Topological restriction of SNARE-dependent membrane fusion. Nature 407, 194198.

Parlati, F., Varlamov, O., Paz, K., McNew, J.A., Hurtado, D., Söllner, T.H., and Rothman, J.E. (2002). Distinct SNARE complexes mediating membrane fusion in Golgi transport based on combinatorial specificity. Proceedings of the National Academy of Sciences 99, 5424-5429.

Pérez-Victoria, F.J., Abascal-Palacios, G., Tascón, I., Kajava, A., Magadán, J.G., Pioro, E.P., Bonifacino, J.S., and Hierro, A. (2010). Structural basis for the wobbler mouse neurodegenerative disorder caused by mutation in the Vps54 subunit of the GARP complex. Proceedings of the National Academy of Sciences 107, 1286012865.

Perez-Victoria, F.J., and Bonifacino, J.S. (2009). Dual roles of the mammalian GARP complex in tethering and SNARE complex assembly at the trans-golgi network. Mol Cell Biol 29, 5251-5263.

Perez-Victoria, F.J., Mardones, G.A., and Bonifacino, J.S. (2008). Requirement of the human GARP complex for mannose 6-phosphate-receptor-dependent sorting of cathepsin D to lysosomes. Mol Biol Cell 19, 2350-2362.

Perez-Victoria, F.J., Schindler, C., Magadan, J.G., Mardones, G.A., Delevoye, C., Romao, M., Raposo, G., and Bonifacino, J.S. (2010). Ang2/fat-free is a conserved subunit of the Golgi-associated retrograde protein complex. Mol Biol Cell 21, 3386-3395. 
Peters, C., Baars, T.L., Buhler, S., and Mayer, A. (2004). Mutual control of membrane fission and fusion proteins. Cell 119, 667-678.

Pfeffer, S.R. (2011). Entry at the trans-face of the Golgi. Cold Spring Harbor perspectives in biology 3, a005272.

Popoff, V., Mardones, G.A., Bai, S.K., Chambon, V., Tenza, D., Burgos, P.V., Shi, A., Benaroch, P., Urbé, S., and Lamaze, C. (2009). Analysis of articulation between clathrin and retromer in retrograde sorting on early endosomes. Traffic 10, 18681880 .

Popoff, V., Mardones, G.A., Tenza, D., Rojas, R., Lamaze, C., Bonifacino, J.S., Raposo, G., and Johannes, L. (2007). The retromer complex and clathrin define an early endosomal retrograde exit site. Journal of cell science 120, 2022-2031.

Posor, Y., Eichhorn-Gruenig, M., Puchkov, D., Schoneberg, J., Ullrich, A., Lampe, A., Muller, R., Zarbakhsh, S., Gulluni, F., Hirsch, E., et al. (2013). Spatiotemporal control of endocytosis by phosphatidylinositol-3,4-bisphosphate. Nature 499, 233237.

Progida, C., and Bakke, O. (2016). Bidirectional traffic between the Golgi and the endosomes - machineries and regulation. J Cell Sci 129, 3971-3982.

Protopopov, V., Govindan, B., Novick, P., and Gerst, J.E. (1993). Homologs of the synaptobrevin/VAMP family of synaptic vesicle proteins function on the late secretory pathway in S. cerevisiae. Cell 74, 855-861.

Robinson, M., Poon, P.P., Schindler, C., Murray, L.E., Kama, R., Gabriely, G., Singer, R.A., Spang, A., Johnston, G.C., and Gerst, J.E. (2006). The Ges1 Arf-GAP mediates Snc1, 2 v-SNARE retrieval to the Golgi in yeast. Molecular biology of the cell 17, 1845-1858.

Robinson, M.S., Sahlender, D.A., and Foster, S.D. (2010). Rapid Inactivation of Proteins by Rapamycin-Induced Rerouting to Mitochondria. Dev Cell 18, 324-331.

Rohn, W.M., Rouillé, Y., Waguri, S., and Hoflack, B. (2000). Bi-directional trafficking between the trans-Golgi network and the endosomal/lysosomal system. J Cell Sci $113,2093-2101$.

Rosa-Ferreira, C., Christis, C., Torres, I.L., and Munro, S. (2015). The small G protein Arl5 contributes to endosome-to-Golgi traffic by aiding the recruitment of the GARP complex to the Golgi. Biology open 4, 474-481.

Rothman, J.E., and Orci, L. (1992). Molecular dissection of the secretory pathway. Nature 355, 409-415. 
Rothman, J.H., and Stevens, T.H. (1986). Protein sorting in yeast: mutants defective in vacuole biogenesis mislocalize vacuolar proteins into the late secretory pathway. Cell 47, 1041-1051.

Rotty, J.D., Wu, C., and Bear, J.E. (2013). New insights into the regulation and cellular functions of the ARP2/3 complex. Nature reviews Molecular cell biology 14, 712.

Sacher, M., Jiang, Y., Barrowman, J., Scarpa, A., Burston, J., Zhang, L., Schieltz, D., Yates, J.R., Abeliovich, H., and Ferro-Novick, S. (1998). TRAPP, a highly conserved novel complex on the cis-Golgi that mediates vesicle docking and fusion. The EMBO Journal 17, 2494-2503.

Schindler, C., Chen, Y., Pu, J., Guo, X., and Bonifacino, J.S. (2015). EARP is a multisubunit tethering complex involved in endocytic recycling. Nat Cell Biol 17, 639-650.

Schmitt-John, T. (2015). VPS54 and the wobbler mouse. Front Neurosci 9, 381.

Schweizer, A., Stahl, P.D., and Rohrer, J. (2000). A di-aromatic motif in the cytosolic tail of the mannose receptor mediates endosomal sorting. J Biol Chem 275, 2969429700 .

Seaman, M.N. (2012). The retromer complex - endosomal protein recycling and beyond. J Cell Sci 125, 4693-4702.

Seaman, M.N., Gautreau, A., and Billadeau, D.D. (2013). Retromer-mediated endosomal protein sorting: all WASHed up! Trends in cell biology 23, 522-528.

Seaman, M.N.J., Michael McCaffery, J., and Emr, S.D. (1998). A Membrane Coat Complex Essential for Endosome-to-Golgi Retrograde Transport in Yeast. The Journal of cell biology 142, 665-681.

Shrivastava, N., Sripada, S., Kaur, J., Shah, P.S., and Cecilia, D. (2011). Insights into the internalization and retrograde trafficking of Dengue 2 virus in BHK-21 cells. PloS one 6 , e25229.

Siniossoglou, S., and Pelham, H.R. (2002a). Vps51p links the VFT complex to the SNARE Tlg1p. The Journal of biological chemistry 277, 48318-48324.

Siniossoglou, S., and Pelham, H.R.B. (2002b). Vps51p Links the VFT Complex to the SNARE Tlg1p. Journal of Biological Chemistry 277, 48318-48324.

Smaczynska-de Rooij, I.I., Allwood, E.G., Aghamohammadzadeh, S., Hettema, E.H., Goldberg, M.W., and Ayscough, K.R. (2010). A role for the dynamin-like protein Vps1 during endocytosis in yeast. J Cell Sci 123, 3496-3506. 
Smaczynska-de Rooij, I.I., Allwood, E.G., Mishra, R., Booth, W.I., Aghamohammadzadeh, S., Goldberg, M.W., and Ayscough, K.R. (2012). Yeast dynamin Vps1 and amphiphysin Rvs167 function together during endocytosis. Traffic 13, 317-328.

Sohda, M., Misumi, Y., Fujiwara, T., Nishioka, M., and Ikehara, Y. (1994). Molecular cloning and sequence analysis of a human 372-kDa protein localized in the Golgi complex. Biochemical and biophysical research communications 205, 1399-1408.

Sztul, E., and Lupashin, V. (2006). Role of tethering factors in secretory membrane traffic. American Journal of Physiology-Cell Physiology 290, C11-C26.

Tie, H.C., Mahajan, D., Chen, B., Cheng, L., VanDongen, A.M., and Lu, L. (2016). A novel imaging method for quantitative Golgi localization reveals differential intra-Golgi trafficking of secretory cargoes. Molecular biology of the cell 27, 848861.

Tokarev, A.A., Alfonso, A., and Segev, N. (2009). Overview of intracellular compartments and trafficking pathways. In Trafficking Inside Cells (Springer), pp. 3-14.

Traub, L.M. (2005). Common principles in clathrin-mediated sorting at the Golgi and the plasma membrane. Biochimica et Biophysica Acta (BBA)-Molecular Cell Research 1744, 415-437.

Ungar, D., Oka, T., Brittle, E.E., Vasile, E., Lupashin, V.V., Chatterton, J.E., Heuser, J.E., Krieger, M., and Waters, M.G. (2002). Characterization of a mammalian Golgi-localized protein complex, COG, that is required for normal Golgi morphology and function. The Journal of cell biology 157, 405-415.

Ungermann, C., and Langosch, D. (2005). Functions of SNAREs in intracellular membrane fusion and lipid bilayer mixing. Journal of Cell Science 118, 38193828 .

van Meer, G., and de Kroon, A.I.P.M. (2011). Lipid map of the mammalian cell. Journal of Cell Science 124, 5-8.

van Meer, G., Voelker, D.R., and Feigenson, G.W. (2008). Membrane lipids: where they are and how they behave. Nat Rev Mol Cell Biol 9, 112-124.

Vardarajan, B.N., Bruesegem, S.Y., Harbour, M.E., Inzelberg, R., Friedland, R., St George-Hyslop, P., Seaman, M.N., and Farrer, L.A. (2012). Identification of Alzheimer disease-associated variants in genes that regulate retromer function. Neurobiol Aging 33, 2231 e2215-2231 e2230.

Watson, P., Jones, A.T., and Stephens, D.J. (2005). Intracellular trafficking pathways and drug delivery: fluorescence imaging of living and fixed cells. Adv Drug Deliv Rev 57, 43-61. 
Whyte, J.R., and Munro, S. (2002). Vesicle tethering complexes in membrane traffic. J Cell Sci 115, 2627-2637.

Willnow, T.E., and Andersen, O.M. (2013). Sorting receptor SORLA-a trafficking path to avoid Alzheimer disease. J Cell Sci 126, 2751-2760.

Witkos, T.M., and Lowe, M. The Golgin Family of Coiled-Coil Tethering Proteins (Front Cell Dev Biol. 2015;3:86. doi:10.3389/fcell.2015.00086.).

Wong, M., and Munro, S. (2014). The specificity of vesicle traffic to the Golgi is encoded in the golgin coiled-coil proteins. Science (New York, NY) 346, 1256898-1256898.

Yip, C.K., Berscheminski, J., and Walz, T. (2010). Molecular architecture of the TRAPPII complex and implications for vesicle tethering. Nature structural \& molecular biology 17, 1298-1304.

Zhang, P., and Schekman, R. (2016). Distinct stages in the recognition, sorting and packaging of proTGF $\alpha$ into COPII coated transport vesicles. Molecular biology of the cell, mbc. E16-02-0090.

Zhao, M., Wu, S., Zhou, Q., Vivona, S., Cipriano, D.J., Cheng, Y., and Brunger, A.T. (2015). Mechanistic insights into the recycling machine of the SNARE complex. Nature 518, 61-67.

Zhong, Q., Lazar, C.S., Tronchère, H., Sato, T., Meerloo, T., Yeo, M., Songyang, Z., Emr, S.D., and Gill, G.N. (2002). Endosomal localization and function of sorting nexin 1. Proceedings of the National Academy of Sciences 99, 6767-6772.

Zolov, S.N., and Lupashin, V.V. (2005). Cog3p depletion blocks vesicle-mediated Golgi retrograde trafficking in HeLa cells. The Journal of cell biology 168, 747-759. 
Table 1: Yeast Strains Used in this Study.

\begin{tabular}{|c|c|c|}
\hline $\begin{array}{l}\text { Strain } \\
\text { Number }\end{array}$ & Source & Genotype \\
\hline KKY 0002 & Invitrogen & MATa his $3 \Delta 1$ leu $2 D$ met $15 \Delta$ ura $3 \Delta$ \\
\hline KKY 0343 & This study & MATa his $3 \Delta$ ura $\Delta l e u \Delta \operatorname{trp} \Delta l y s \Delta$ \\
\hline KKY 0352 & This study & MATa his $3 \Delta u r a \Delta l e u \Delta t r p \Delta l y s \Delta$ VPS1::KanMx6 \\
\hline KKY 0815 & This study & KKY 343 VPS51-GFP-HIS3 \\
\hline KKY 0828 & This study & KKY 352 VPS51-GFP-HIS3 \\
\hline KKY 0995 & This study & KKY 352 (pRS416-GFP-Snc1-PEM) \\
\hline KKY 0999 & This study & KKY 002 SEC7-RFP-KanMx6 \\
\hline KKY 1002 & This study & KKY 352 SEC7-RFP-KanMx6 \\
\hline KKY 1004 & This study & KKY 002 (pRS416-GFP-Snc1-PEM) \\
\hline KKY 1254 & Clontech & $\begin{array}{l}\text { MATa, trp1-901, leu2-3, 112,ura3-52, his3-200, gal4A, } \\
\text { gal804,LYS2 }: \because \text { GAL1UAS-Gal1TATA-His3, GAL2UAS- } \\
\text { Gal2TATA-Ade2 URA3 :: MEL1UAS-Mel1TATAAUR1-C } \\
\text { MEL1 }\end{array}$ \\
\hline KKY 1255 & Clontech & 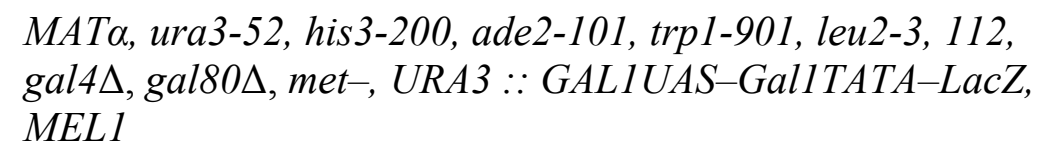 \\
\hline KKY 1272 & This study & KKY 1254 (pGBKT7-LAM) \\
\hline KKY 1273 & This study & KKY $1255(p G A D T 7-T)$ \\
\hline KKY 1274 & This study & KKY 1254 (pGBKT7-53) \\
\hline KKY 1275 & This study & KKY 1254 (pGBKT7-VPSI) \\
\hline KKY 1304 & This study & KKY 1274/KKY 1273 (diploid) \\
\hline KKY 1305 & This study & KKY 1272/KKY 1273 (diploid) \\
\hline KKY 1438 & This study & KKY 1254 (pGBKT7-Vps1-GTPase) \\
\hline KKY 1439 & This study & KKY 1254 (pGBKT7-Vps1-Middle) \\
\hline KKY 1440 & This study & KKY 1254 (pGBKT7-Vps1-GED) \\
\hline KKY 1503 & This study & KKY 1275/KKY 1513 (diploid) \\
\hline KKY 1504 & This study & KKY 1275/KKY 1830 (diploid) \\
\hline KKY 1505 & This study & KKY 1275/KKY 1831 (diploid) \\
\hline KKY 1506 & This study & KKY 1275/KKY 1831(diploid) \\
\hline
\end{tabular}


Table 1. Yeast Strains Used in this Study (continued)

\begin{tabular}{|c|c|c|}
\hline $\begin{array}{l}\text { Strain } \\
\text { Number }\end{array}$ & Source & Genotype \\
\hline KKY 1507 & This study & 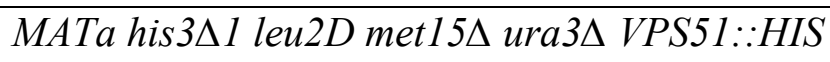 \\
\hline KKY 1513 & This study & KKY 1255 (pGADT7-Vps51) \\
\hline KKY 1525 & This study & KKY 002 (pKT1490-GFP-Snc1) \\
\hline KKY 1526 & This study & KKY 352 (pKT1490-GFP-Snc1) \\
\hline KKY 1579 & This study & KKY 815 (pRS416-mRFP-Vps1) \\
\hline KKY 1595 & This study & KKY 1507 (pKT1490-GFP-Snc1) \\
\hline KKY 1672 & This study & KKY 815 (pRS426-Gga1-mRFP) \\
\hline KKY 1673 & This study & KKY 828 (pRS426-Gga1-mRFP) \\
\hline KKY 1697 & This study & KKY 1526 (pRS426-Vps51-mRFP) \\
\hline KKY 1698 & This study & KKY 1595 (pRS416-mRFP-Vps1) \\
\hline KKY 1750 & This study & KKY 1255 (pGADT7-Vps51 (1-132)) \\
\hline KKY 1752 & This study & KKY 1438/KKY 1750 (diploid) \\
\hline KKY 1753 & This study & KKY 1439/KKY 1750 (diploid) \\
\hline KKY 1754 & This study & KKY 1440/KKY 1750 (diploid) \\
\hline KKY 1777 & This study & KKY 1255 (pGADT7-Vps51 $\left.1^{E 127 K}\right)$ \\
\hline KKY 1778 & This study & KKY 1255 (pGADT7-Vps51 $\left.1^{Y 129 F}\right)$ \\
\hline KKY 1779 & This study & KKY 1255 (pGADT7-Vps51 \\
\hline KKY 1780 & This study & KKY 1275/KKY 1777 (diploid) \\
\hline KKY 1781 & This study & KKY 1275/KKY 1778 (diploid) \\
\hline KKY 1782 & This study & KKY 1275/KKY 1779 (diploid) \\
\hline KKY 1783 & This study & KKY 999 (pKT1566-GFP-Tlg1) \\
\hline KKY 1784 & This study & KKY 1002 (pKT1566-GFP-Tlg1) \\
\hline KKY 1796 & This study & KKY 1507 (pRS426) \\
\hline KKY 1797 & This study & KKY 1507 (pRS426-Vps51-mRFP) \\
\hline KKY 1798 & This study & KKY 1507 (pRS426-Vps51 $\left.1^{E 127 K}-m R F P\right)$ \\
\hline KKY 1799 & This study & KKY 1507 (pRS426Vps5 $\left.51^{Y 129 F}-m R F P\right)$ \\
\hline KKY 1800 & This study & KKY 1507 (pRS426-Vps51EY127, 129KF $-m R F P)$ \\
\hline
\end{tabular}


Table 1. Yeast Strains Used in this Study (continued)

\begin{tabular}{|c|c|c|}
\hline $\begin{array}{l}\text { Strain } \\
\text { number }\end{array}$ & Source & Genotype \\
\hline KKY 1817 & This study & KKY 1507 (pRS416-GFP-Sncl-PEM) \\
\hline KKY 1822 & This study & KKY 1595 (pRSVps51-mRFP) \\
\hline KKY 1823 & This study & KKY 1595 (pRS426-Vps51 $\left.1^{E 127 K}-m R F P\right)$ \\
\hline KKY 1824 & This study & KKY 1595 (pRS426-Vps51 \\
\hline KKY 1825 & This study & KKY 1595 (pRS426-Vps51 \\
\hline KKY 1826 & This study & KKY 1797 (pKT1560-GFP-Rcyl) \\
\hline KKY 1827 & This study & KKY 1798 (pKT1560-GFP-Rcyl) \\
\hline KKY 1828 & This study & KKY 1799 (pKT1560-GFP-Rcyl) \\
\hline KKY 1829 & This study & KKY 1800 (pKT1560-GFP-Rcyl) \\
\hline KKY 1830 & This study & KKY 1255 (pGADT7-Vps52) \\
\hline KKY 1831 & This study & KKY 1255 (pGADT7-Vps53) \\
\hline KKY 1832 & This study & KKY 1255 (pGADT7-Vps54) \\
\hline
\end{tabular}


Table 2. Bacterial Plasmids Used in this Study

\begin{tabular}{lll}
\hline Plasmid Number & Plasmid Name & Source \\
\hline KKD 0003 & pFA6Aa-KanMx6 & (Longtine et al., 1998) \\
KKD 0005 & pFA6a-His3Mx6 & (Longtine et al., 1998) \\
KKD 0008 & pFA6a-GFP-HIS & (Longtine et al., 1998) \\
KKD 0022 & mRFP-KAN & (Longtine et al., 1998) \\
KKD 0062 & pRS416-GFP-Snc1-PEM & (Furuta et al., 2007) \\
KKD 0064 & pKT1490-GFP-Snc1 & (Furuta et al., 2007) \\
KKD 0067 & pKT1560-GFP-Rcy1 & (Furuta et al., 2007) \\
KKD 0069 & pKT1566-GFP-Tlg1 & (Furuta et al., 2007) \\
KKD 0079 & pGBKT7-Vps1 & This study \\
KKD 0083 & pGADT7 & Clontech \\
KKD 0085 & pGADT7-T & Clontech \\
KKD 0099 & pGBKT7 & Clontech \\
KKD 0100 & pGBKT7-53 & Clontech \\
KKD 0101 & pGBKT7-Lam & Clontech \\
KKD 0127 & pRS426 & Phil Hieter \\
KKD 0128 & pGBKT7-Vps1-MID & This study \\
KKD 0130 & pGBKT7-Vps1-GED & This study \\
KKD 0134 & pGBKT7-Vps1-GTPase & This study \\
KKD 0143 & pRS416-mRFP-Cps1 & (Obara et al., 2013) \\
KKD 0156 & pGADT7-Vps51 & This study \\
KKD 0158 & pGADT7-Vps53 & This study \\
KKD 0159 & pGADT7-Vps54 & This study \\
KKD 0162 & pGADT7-Vps52 & This study \\
KKD 0190 & pRS416-mRFP-Vps1 & This study \\
KKD 0248 & pGADT7-Vps51 (1-33) & This study \\
KKD 0249 & pGADT7-Vps51 (1-66) & This study \\
KKD 0250 & pGADT7-Vps51 (1-132) & This study \\
KKD 0261 & This study \\
KKD 0298 & This study \\
\hline
\end{tabular}


Table 2. Bacterial plasmids used in this study (continued)

\begin{tabular}{|c|c|c|}
\hline Plasmid Number & Plasmid Name & Source \\
\hline KKD 0321 & pGADT7-Vps51 ${ }^{\text {E127K }}$ & This study \\
\hline KKD 0328 & pGADT7-Vps51 ${ }^{\mathrm{Y} 129 \mathrm{~F}}$ & This study \\
\hline KKD 0329 & pGADT7-Vps51 ${ }^{\text {EY127, 129KF }}$ & This study \\
\hline KKD 0330 & pRS426-Vps51 ${ }^{\mathrm{E} 127 \mathrm{~K}}-\mathrm{mRFP}$ & This study \\
\hline KKD 0331 & pRS426-Vps51 ${ }^{\mathrm{Y} 129 \mathrm{~F}}-\mathrm{mRFP}$ & This study \\
\hline KKD 0332 & pRS426-Vps51 $1^{\mathrm{EY} 127,129 \mathrm{KF}}-\mathrm{mRFP}$ & This study \\
\hline
\end{tabular}




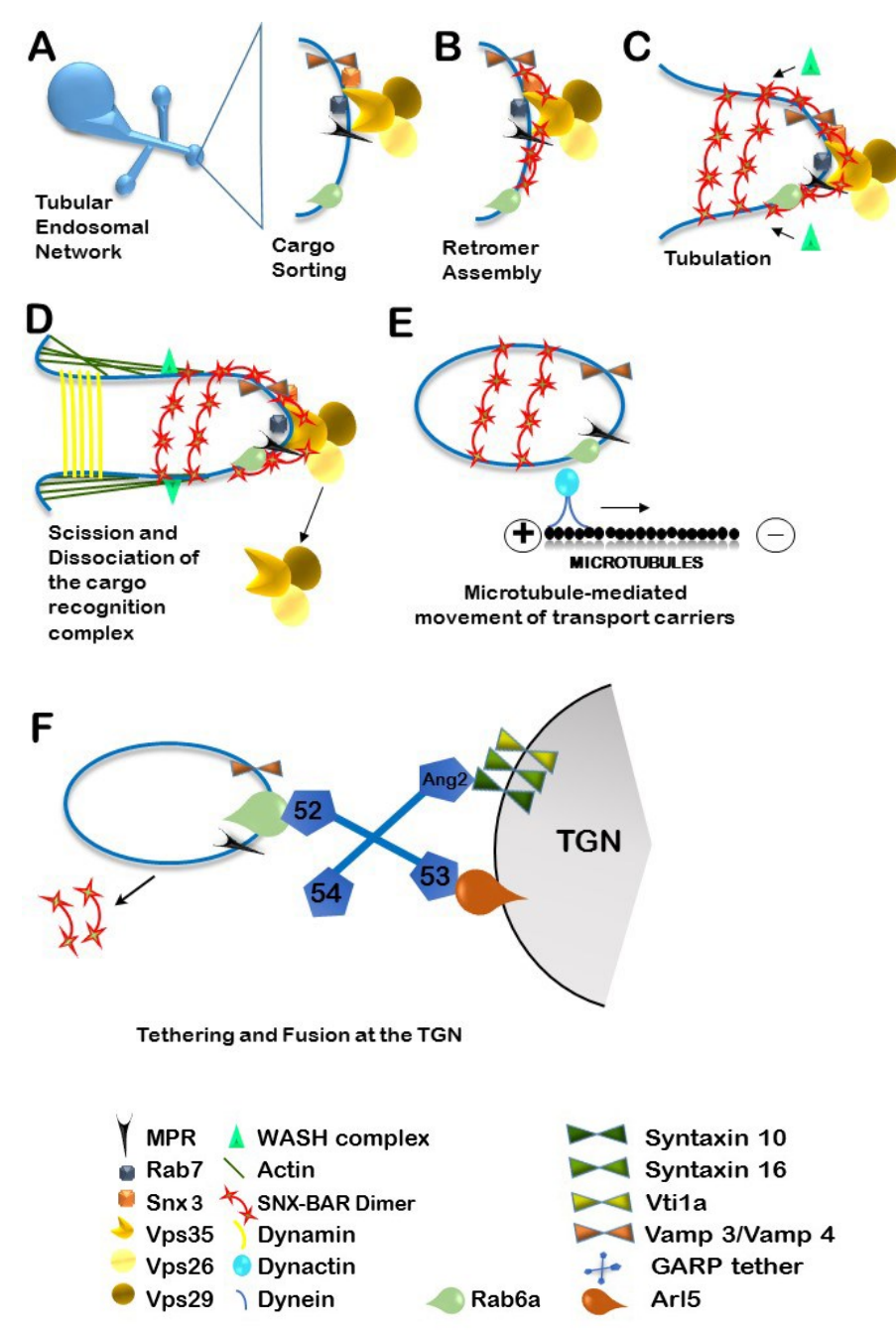

Figure 1. Retrograde transport from the endosome to the TGN. A) The cargo recognition subcomplex of the retromer is recruited to the endosomal membrane by Rab7 and Snx3. The retromer subunit Vps35 recognizes the WLM motif on the cytosolic tail of the MPR and binds to it. B) The SNX-BAR subcomplex recognizes the high PI3P concentration of the endosomal membrane, binds to it, and initiates tubule formation. C) As tubulation proceeds, the WASH complex is recruited by Vps35 to the neck of the extending tubule to stimulate actin polymerization. D) Actin polymers push the extending tubule away from the endosomal membrane, into the cytosol. Meanwhile, dynamin is recruited to the neck of the tubule, and it pinches the tubule off by utilizing the energy from GTP hydrolysis. Once the tubule is pinched off, the cargo recognition subcomplex dissociates from the cargo and returns to the endosomal membrane. E) The sorting nexins interact with dynactin which is associated with dynein, a minus-end molecular motor. The dynein-dynactin motor pulls the cargo-laden transport carrier along microtubule tracks towards the TGN. F) Upon reaching the proximity of the TGN, the sorting nexins dissociate and recycle to the TEN. The GARP tether is recruited by the GTPase Rab6a, and different components of the tether interact with both the transport carrier and the TGN. Finally, the $t$-SNAREs and $v$-SNARE associate and bridge the transport carrier to the TGN, enabling membrane fusion and cargo transfer to the TGN (Seaman et al., 2013). 

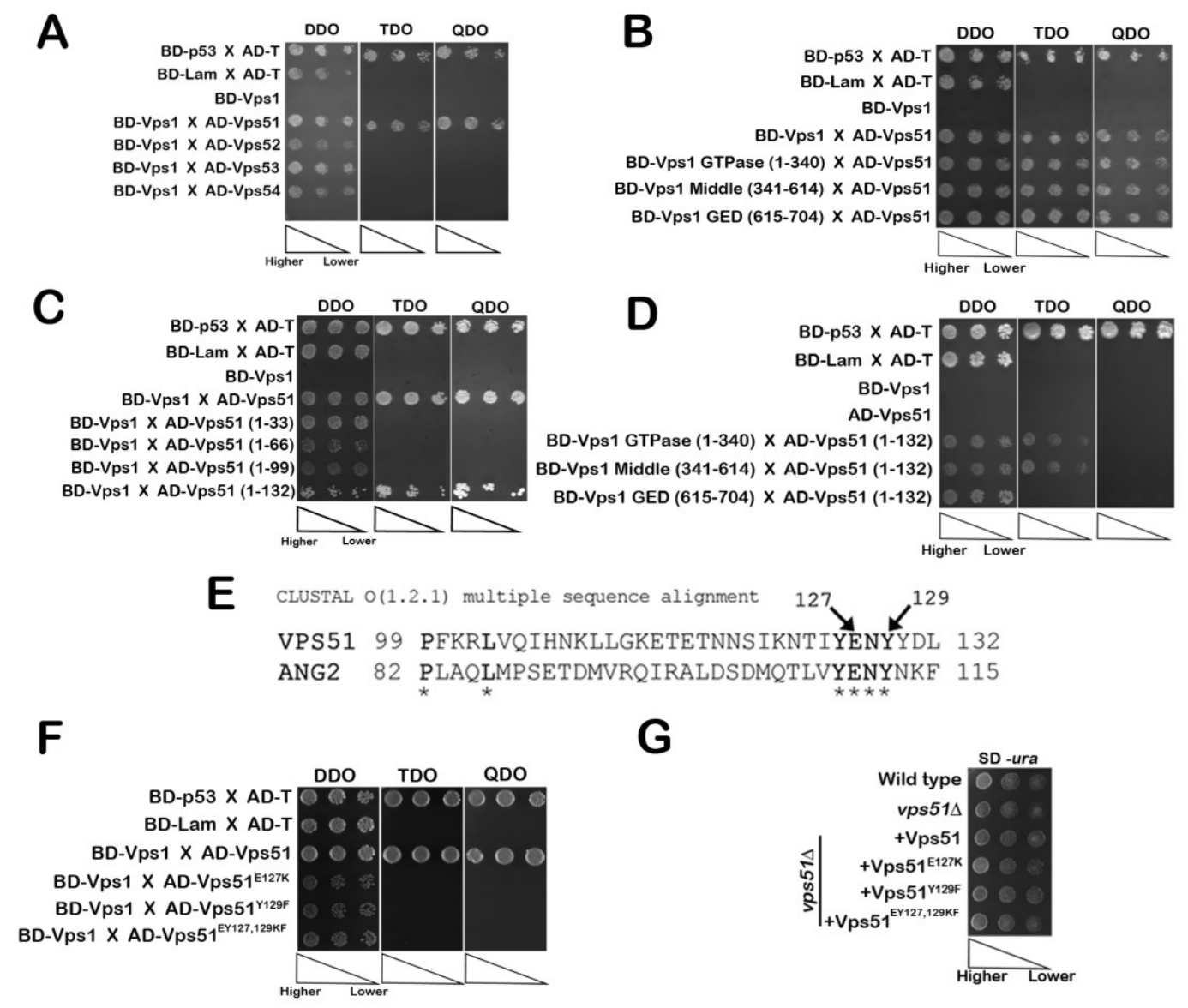

Figure 2. Characterization of the interaction between Vps1 and Vps51. A) Diploid strains coexpressing BD-Vps1 and an AD fused GARP subunit (Vps51, Vps52, Vps53 or Vps54) were made, and subjected to a spotting assay. A strain co-expressing BD-p53 and AD-SV40 large T antigen (AD-T) (KKY 1304) was used as a positive control. Another strain co-expressing BDLamin and AD-T (KKY 1305) was used as a negative control. A strain expressing only BD-Vps1 (KKY 1275) was utilized as an additional negative control. These cells were diluted by a factor of 3 and spotted onto DDO, TDO, and QDO plates. B) Three individual strains were engineered to express a BD fused domain of Vps1: the N-terminal GTPase domain (1-340 aa) (KKY 1438), the Middle domain (341-614 aa) (KKY 1439) or the C-terminal GED domain (615-704 aa) (KKY 1440). These strains were mated with a strain expressing AD-Vps51 (KKY 1513) and spotted. C) A series of $\mathrm{AD}$ fused C-terminal truncations of $\mathrm{Vps} 51$ were co-expressed with $\mathrm{BD}-\mathrm{Vps} 1$, and subjected to a spotting assay. D) BD fused domains of Vps1 and AD-Vps51 (1-132 aa) (KKY1750) were co-expressed in yeast cells and spotted onto DDO, TDO, and QDO. E) Sequences of both Vps51 and its mammalian homolog ANG2 were aligned using CLUSTAL OMEGA tool and the residues that were $100 \%$ conserved are indicated using asterisks. The residues that were eventually mutated are shown with arrows. F) BD-Vps1 was co-expressed with an AD fused Vps51 mutant: Vps51 ${ }^{\mathrm{E} 127 \mathrm{~K}}$ (KKY 1780), Vps51 ${ }^{\mathrm{Y} 129 \mathrm{~F}}$ (KKY 1781), or Vps51 ${ }^{\mathrm{EY} 127,}$ ${ }_{129 \mathrm{KF}}$ (KKY 1782), and tested for interaction. G) Both point mutants Vps51 ${ }^{\mathrm{E} 127 \mathrm{~K}}$ and Vps51 ${ }^{\mathrm{Y} 129 \mathrm{~F}}$ and the double mutant were introduced into vps51 $1 \Delta$ creating KKY 1808, 1809 and 1810. These new strains along with adequate controls were spotted onto SD (-Ura) plates. For all data shown in this figure, the concentration gradient is denoted by the triangle with its tip pointing towards the lower concentration. 


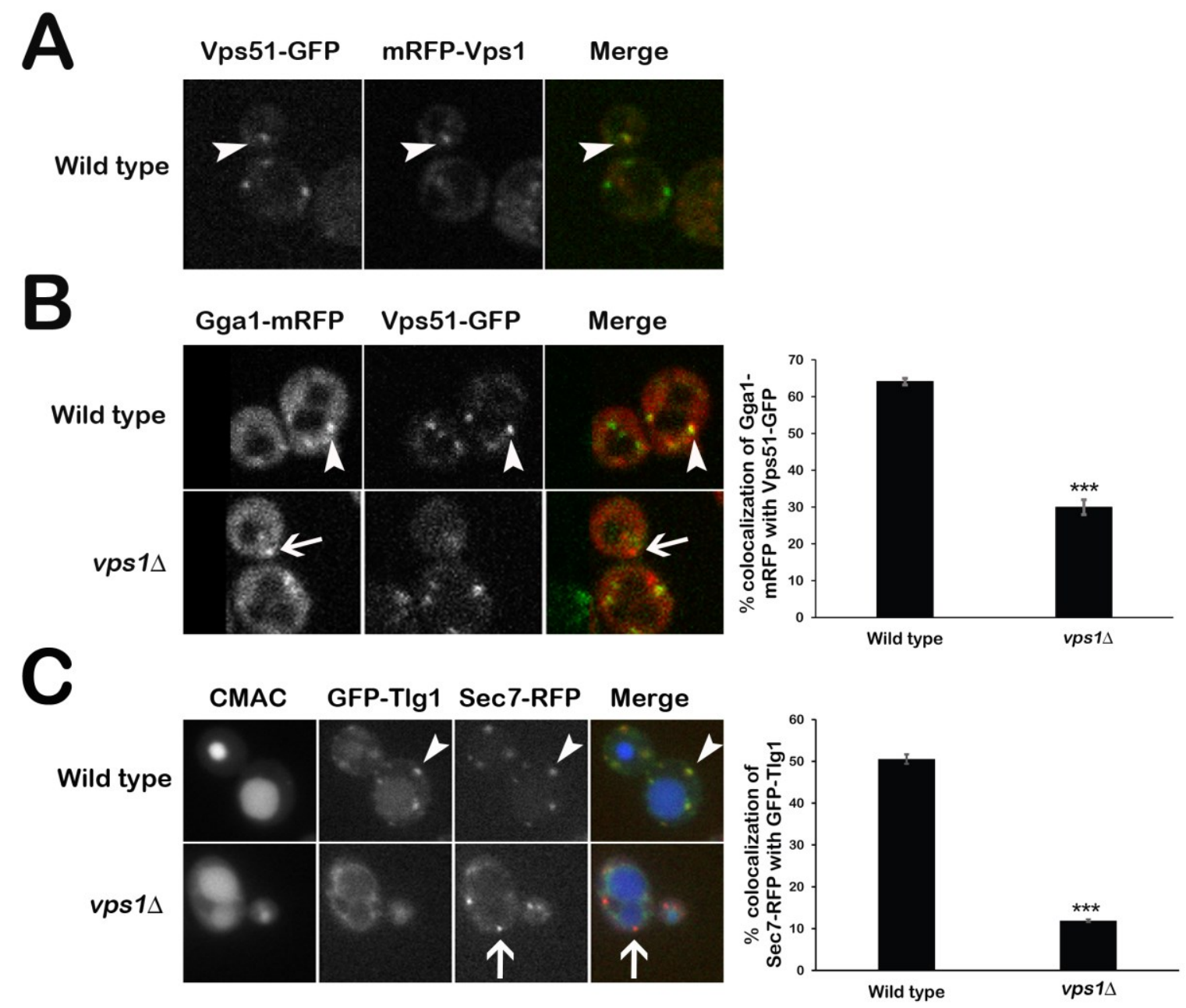

Figure 3. Subcellular dynamics of Vps1 and Vps51. A) Both mRFP-Vps1 and Vps51-GFP were co-expressed in a wild-type cell (KKY 1579), and confocal microscopy was used to determine the presence of colocalization. For the purpose of this study, colocalization was defined as yellow puncta appearing due to spatial overlap of red and green puncta. B) Gga1-mRFP and Vps51-GFP were both expressed in wild type and vps $1 \Delta$ cells and the extent of colocalization of Ggal-mRFP puncta with Vps51-GFP puncta was determined. C) Wild type and $v p s 1 \Delta$ cells were made to coexpress GFP-Tlg1 and Sec7-RFP. These modified strains were then stained with Cell TrackerTM CMAC. The degree of colocalization of Sec7-RFP with Tlg1-GFP was determined. In all the images shown above, the arrowheads indicate colocalizing puncta, and the arrows indicate puncta that do not colocalize. The quantitation shown is an average of three trials $(n=30$ cells). 


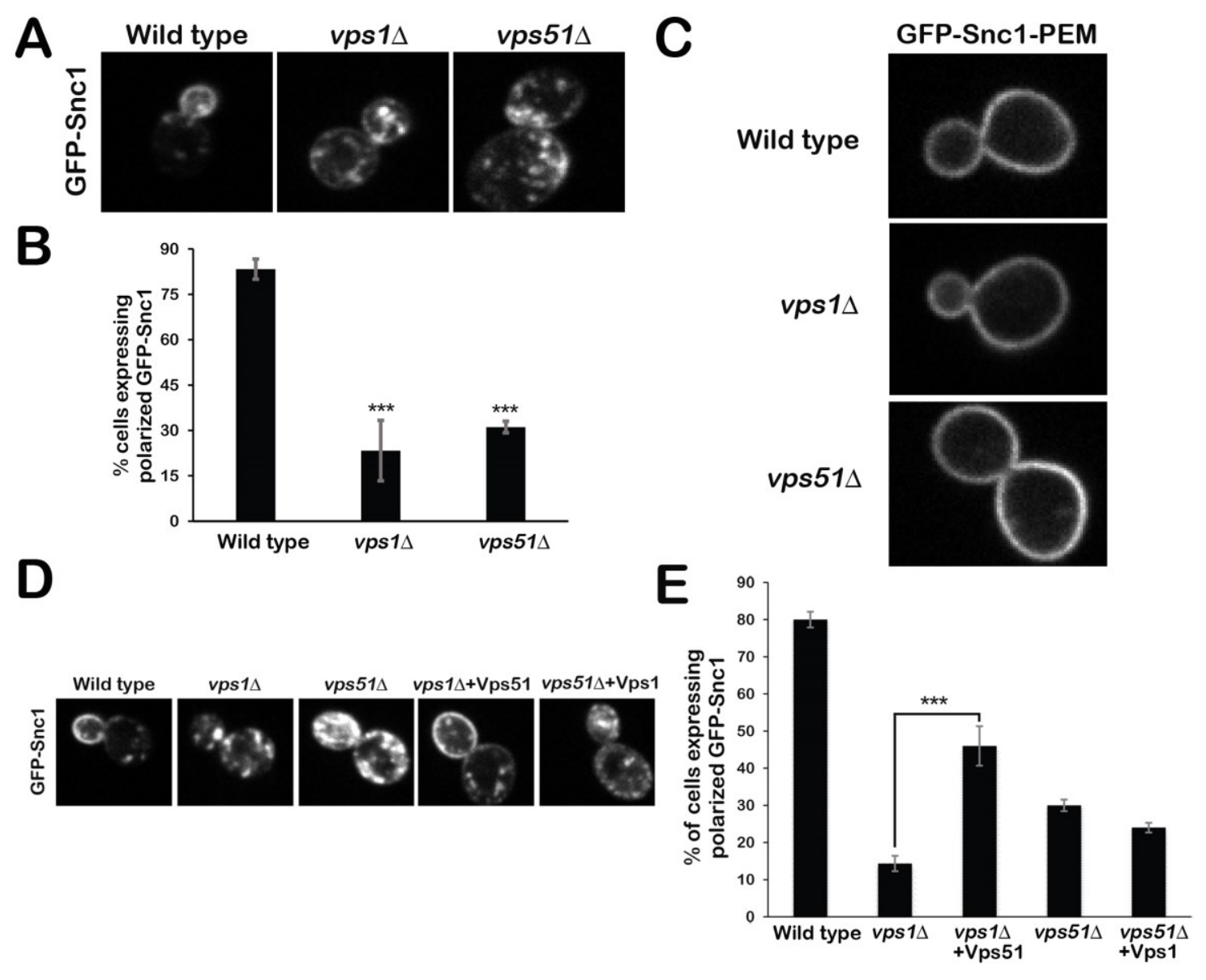

Figure 4. Vps1 and Vps51 collaborate for retrograde transport of $v$-SNARE Snc1. A) GFP-Snc1 was exogenously expressed in wild type, $v p s 1 \Delta$, and $v p s 51 \Delta$ strains, and a polarized appearance of GFP-Snc1 was considered as the proper targeting of Snc1. B) The percentage of cells with GFP-Snc1 found at the bud plasma membrane was quantified. Statistical significance was estimated using the student's T test and represented as p-values. P-values less than or equal to 0.001 was indicated with three asterisks, $p$-values less than or equal to 0.01 was indicated with two asterisks, and p-values less than or equal to 0.05 was indicated with an asterisk. C) An endocytosis-defective mutant of Snc1 (GFP-Snc1-PEM) was expressed in the same strains as in F3A and visualized. D) Vps1 was overexpressed in a vps51 strain. Similarly, Vps51 was overexpressed in a vps $1 \Delta$ strain. Levels of Sncl polarization in these strains were examined using confocal microscopy. E) Percentage of polarized cells was determined for each strain used in F4D. 

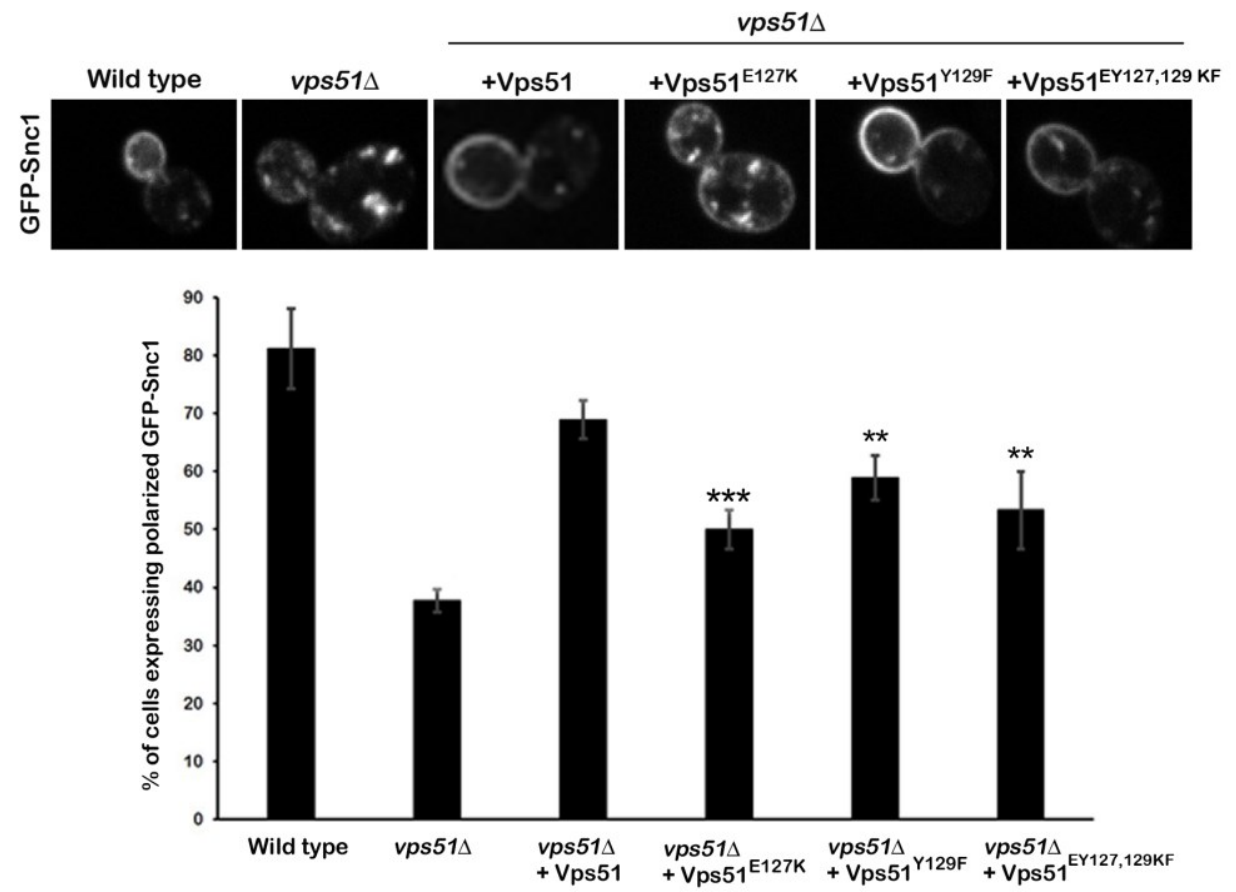

Figure 5. Physiological significance of Vps1-Vps51 interaction. Representative images of GFPSnc1 localization in cells expressing Vps51 point mutant species (Vps51 ${ }^{\mathrm{E} 127 \mathrm{~K}}, \mathrm{Vps} 51^{\mathrm{Y} 129 \mathrm{~F}}$, or Vps51 ${ }^{\mathrm{EY} 127,129 \mathrm{KF}}$ ) is shown. Levels of GFP-Snc1 polarization in vps 51 mutant cells was determined. All quantification shown in this figure is an average of three individual data sets $(n=30$ cells $)$. 


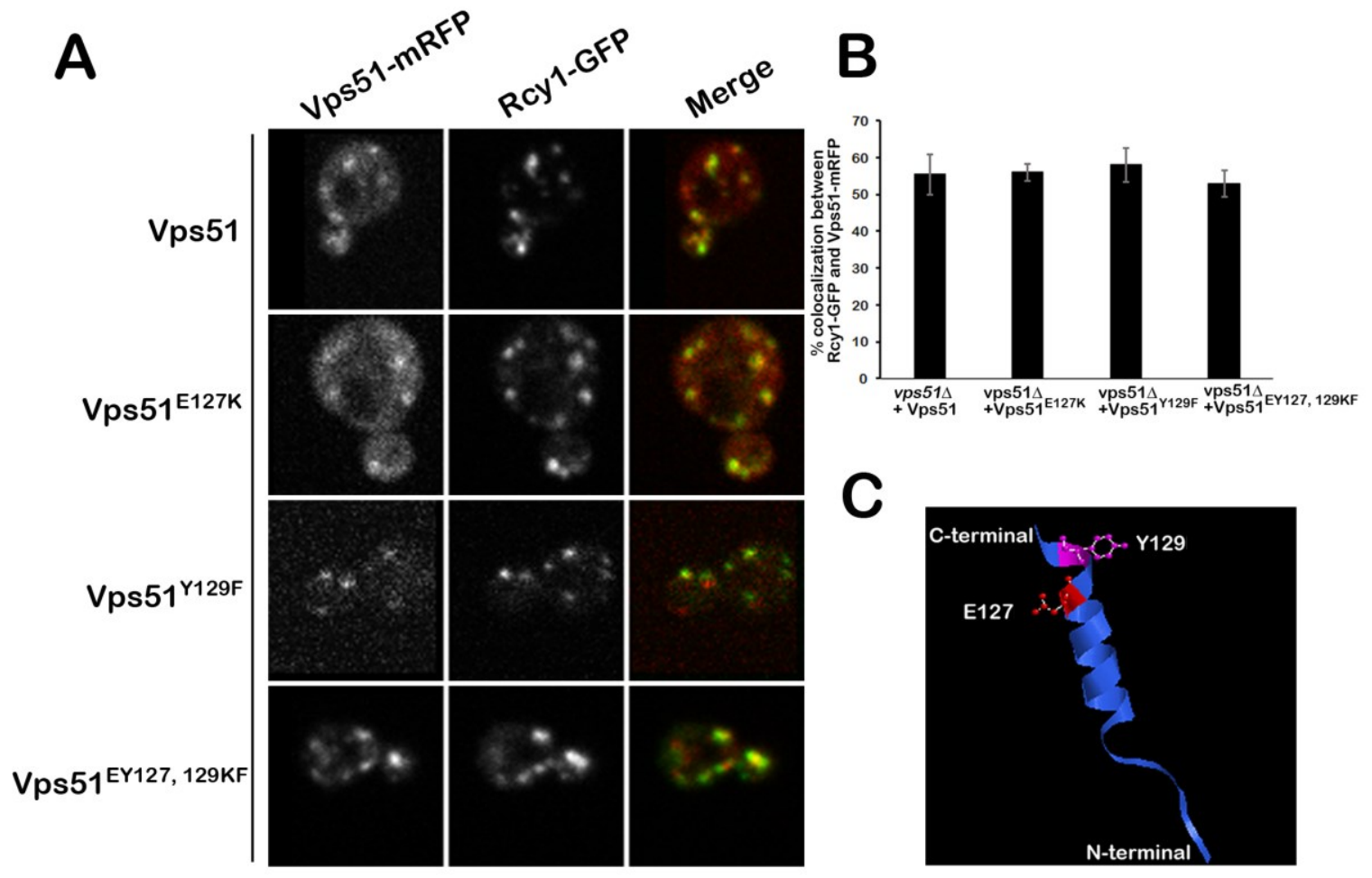

Figure 6. Effect of the point mutations on the localization of Vps51. A) Rcy1-GFP was coexpressed with Vps51-mRFP or an indicated mutant copy of Vps51-mRFP. Confocal microscopy was used to determine colocalization. B) A homology model of Vps51 based on Ang2 was constructed using the SWISSPROT and RasTop tools. The residues E127 and Y129 are indicated in the model. 


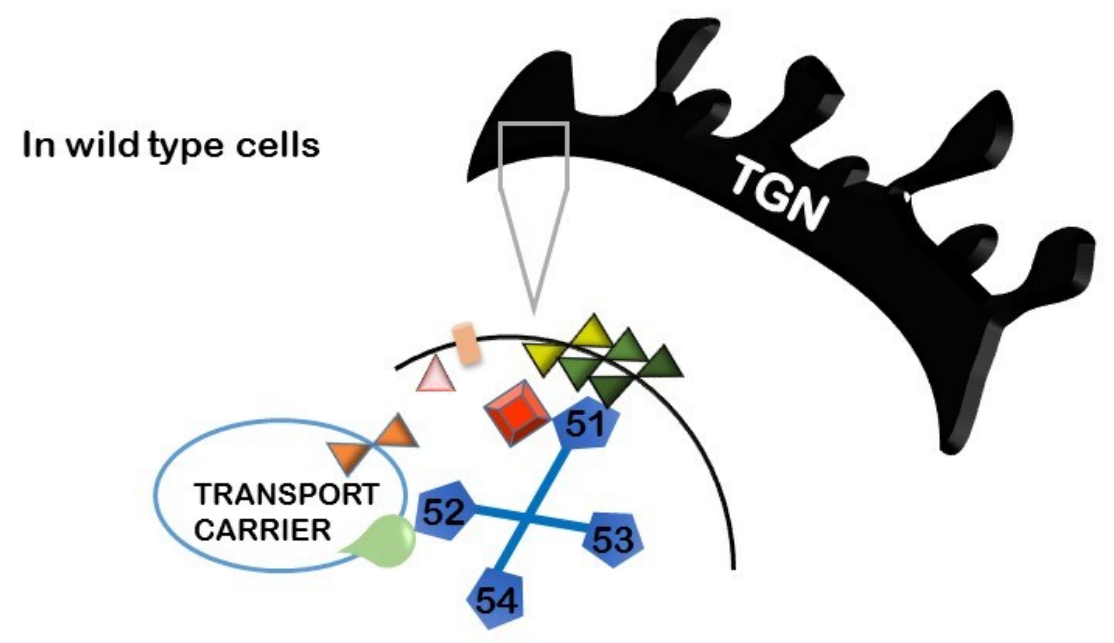

In vps $1 \Delta$ cells
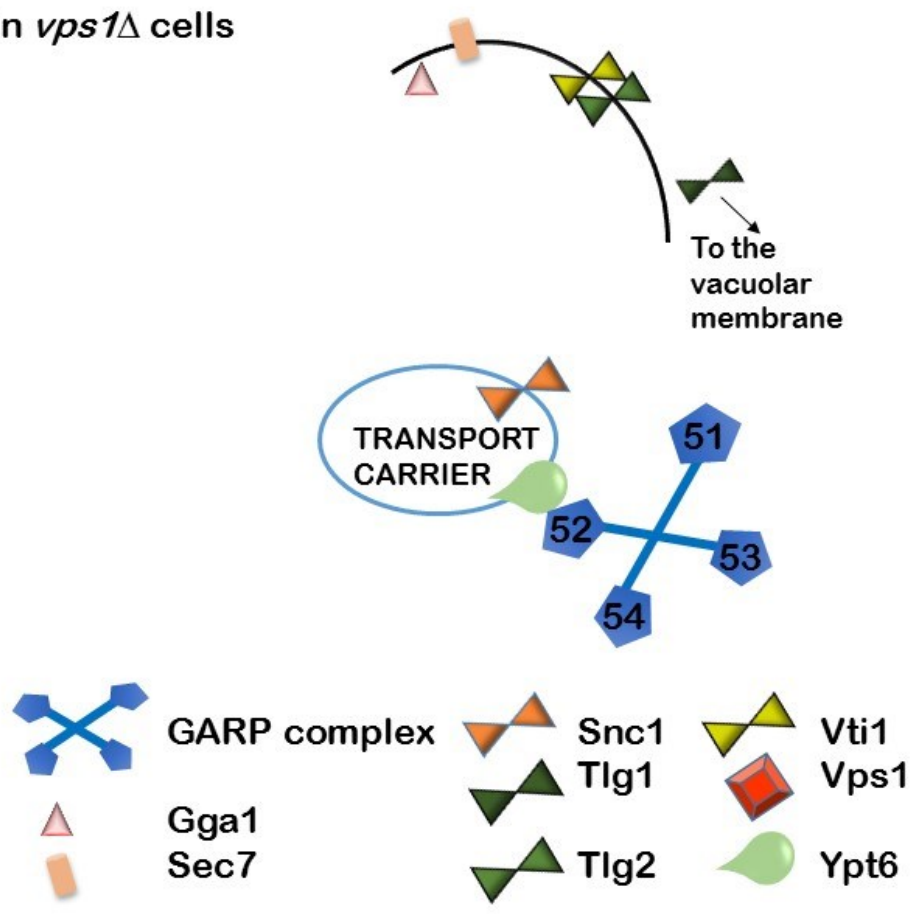

Figure 7. Model for tethering/fusion at the TGN depicting the functional protein network in a wild type and vps $1 \Delta$ background. Under normal conditions, the cargo-enriched transport carrier is captured by the GARP tether subunit Vps52 through the interaction with Ypt6, a yeast homologue of Rab6a. Vps51, another subunit of the GARP, interacts with Tlg1, a Golgi $t$ SNARE to anchor the transport carrier at the TGN. Finally, three $t$-SNAREs (Tlg1, Tlg2, and Vti1) and a $v$-SNARE (Snc1) associate to form trans-SNARE complex, enabling membrane fusion and cargo transfer to the TGN. In the absence of Vps1, colocalization levels of Vps51 and Tlg1 with the late Golgi are signifcantly diminished. As a result, the Ypt6-Vps52-Vps51-Tlg1 connection is compromised, thereby negatively affecting the tethering/fusion activity occuring at the face of the late Golgi. 